\title{
5 Flow Measurements
}

\subsection{Introduction}

This chapter will include current meters used in environmental sciences and industrial flowmeters because they share many common characteristics and it was considered satisfactory to present them together. Orifice plate, Venturi, Pitot, Annubar and positive-displacement flowmeters are not included because they are traditional flowmeters that may be found in many books (Miller, 1996).

In general, the operating principle is the same whether the instrument is used in industry or in science and they have constructive differences only, as it is the measuring range. Usually industrial speeds are higher than current velocities in natural environments. Another difference is that in industrial applications, most of the times flows are measured along only one direction. Instead, in environmental sciences the velocity of the current is required along two or three orthogonal directions.

In industrial instruments, designers do not have to care about the amount of energy required to power them because they are connected to the main alternating voltage power supply so they can employ large signals to obtain a good signal to noise ratio. This is necessary to get high reliable measurements because industrial environments are usually noisy, from an electrical point of view.

Industrial flowmeters may be used to evaluate the amount of fluid product that an industry is transferring to another through a pipe, and the billing depends on the flowmeter measurements. Then these flowmeters have to be very reliable and stable, normally, more than those current meters used for research applications. Typically, each of the industries has its own flowmeter on the same pipe and both meters should measure the same quantity with errors below $0.5 \%$.

Environmental scientific research instruments are usually powered by batteries, so power availability constrains their design, giving as a result low exciting voltages and currents, which could generate weak signals. Fortunately, electrical noise in nature is generally much less than in industries, and a good signal to noise relation can be achieved even with low power supply.

The overall consequence of the above-mentioned differences is that an instrument designed for industrial use cannot be used in field research and vice-versa, but from a didactic point of view it is convenient to treat them together; starting with the description of the operating principles, noting the differences in the way they are constructed and showing similarities in the specifications. This strategy is applicable to instruments based on electromagnetic, acoustic and thermal working principles. There are, however, some technologies that have been developed for measuring only inside pipes, and so far they have not been used for measuring in open channels; one of them is the Coriolis mass flowmeter. 
When trying to measure flow in industry, it is important to note that industrial processes very often require measuring the mass flow rate. The interest in knowing the mass transfer is because the revenue and some actions in automatic control are associated to the mass. For example, the amount of energy of fuel is associated to its mass. Also, in automatic chemical processes where different quantities of products have to be mixed, the equations that govern the mix are generally expressed in amounts of mass. Some flow meters such as those based on the Coriolis force and thermal transportation measure mass flow rate directly.

Other flowmeters, such as those based on acoustics, vortex, electromagnetism, etc., measure the speed of the fluid, which associated to the pipe cross sectional area gives the volumetric flow rate. Volumetric flowmeters can obtain the mass flow rate as an indirect measure by introducing the density of the fluid.

\subsection{Vortex Flowmeter}

These flowmeters base their operation in the existence of vortices in the fluid where flow is to be measured. A vortex is an eddy or swirl of fluid. The flutter of a flag, the whistle of the wind in the branches of trees and eddies at the stern of moving boats are common examples of vortices.

If an object is moved in a static fluid, vortices are formed within the fluid. If a fluid moves inside a pipe, vortices can be generated by placing an object with a flat front as shown in Figure 5.1. This object is a non-streamlined body called a "shedder bar" and the phenomenon is called vortex shedding.

In vortex flowmeters it is required to generate vortices by a shedder bar. The flowmeter manufacturer adopts different shapes for the bar, which is often installed vertically in the pipe (it is shown horizontally in Figure 5.1 to make the picture clearer).

Under turbulent conditions fluid cannot follow the shape of the vortex generator and it is separated from the bar forming two wakes. Vortices are areas where fluid speed is higher and pressure is lower than in the surrounding fluid. They rotate clockwise in one wake and counterclockwise in the other. Vortices are formed one at a time in alternating wakes, resulting in an oscillating pressure difference (Kármán vortex street). Usually piezoelectric sensors are placed downstream to detect this fluctuating pressure gradient.

According to Eq. (5.1), as fluid velocity $(V)$ increases, so does vortex frequency $(f)$,

$$
f=S t \frac{V}{b} ; V=\frac{f_{b}}{S t}
$$

where $b$ is the shedder bar width and $S t$ is a dimensionless number called the Strouhal number. A calibration process allows $S t$ to be found for a certain shedder bar shape. Fluid velocity can then be calculated by measuring the vortex frequency. 
Piezoelectric

Pipe section
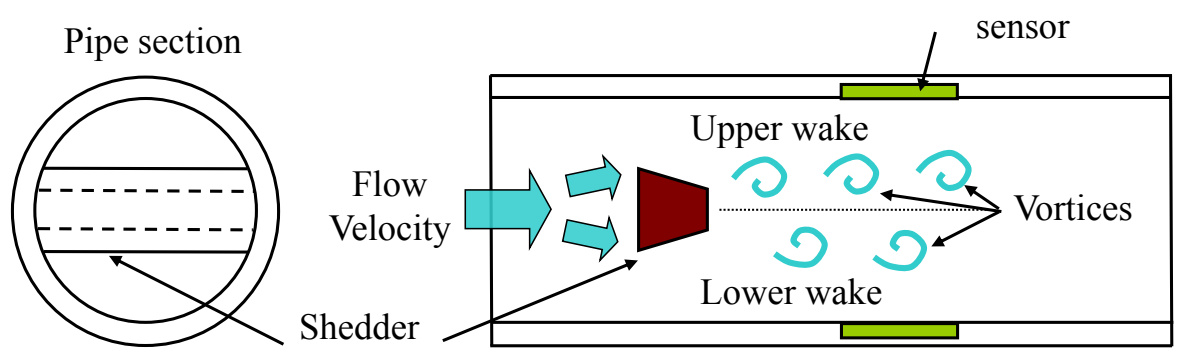

bar

Fig. 5.1: Sectional view of a Vortex flowmeter. The shedder bar generates alternating vortices which are detected by means of piezoelectric sensors.

Since the Strouhal number may vary for low Reynolds numbers, flowmeters should be used in ranges where $S t$ is constant. Figure 5.2 shows $S t$ as a function of the Reynolds number $(\mathrm{Re})$. The linear zone in this picture depends on the vortex generator shape. Today's flowmeters, which have solid state memory and intelligent processors, are able to correct the transfer function, extending their useful range down.

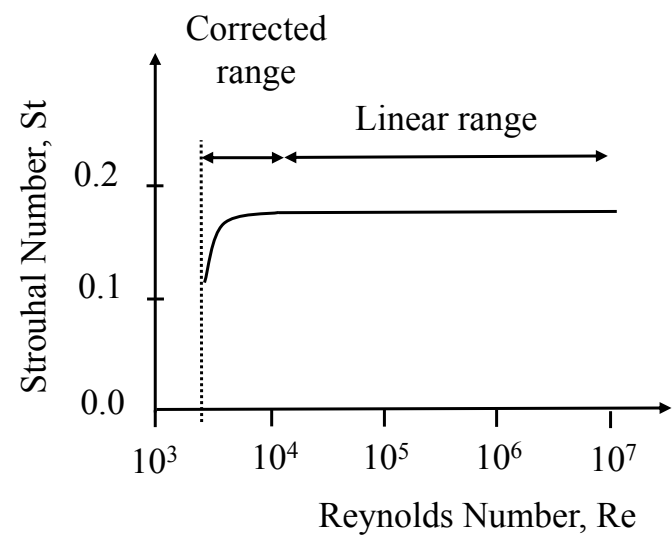

Fig. 5.2: Strouhal number as a function of Reynolds number. Nowadays instruments are able to correct the transfer function in the non linear lower range.

Some manufacturers also measure temperature $(T)$ and pressure $(P)$. Then using equations stored in the flowmeter memory they are able to calculate the fluid density and viscosity, the Reynolds number (to select the correction factor for low-velocity fluids) and the volumetric and mass flow rates.

In addition to the piezoelectric sensors, there are other ways of detecting vortex frequency which are based on capacitive, thermal and acoustic sensors. 


\subsubsection{Vortex Flowmeter Characteristics}

Vortex flowmeters can be used with gases, liquids and steam. Some do not allow measuring bidirectional flows. They are sensitive to distortions of the speed profile, and then stable flow conditions upstream are required. The presence of the shedder bar results in pressure losses that are about half of that produced by an orifice plate flow meter. Excessive wear or corrosion in the shedder bar can change the calibration constant.

Vortex flowmeters can be used in dynamic ranges of 1:10 for liquids and 1:20 for gases, and can measure up to about $10 \mathrm{~m} / \mathrm{s}$ in liquids, and $100 \mathrm{~m} / \mathrm{s}$ in gases (see Section (2.2.1) for dynamic range definition). In general, viscous fluids cannot be measured due to their low Reynolds number.

\subsection{Electromagnetic Flowmeters}

\subsubsection{Principle of Operation}

The principle of operation of an electromagnetic flowmeter is based on Faraday's law, and is shown schematically in Figure 5.3. A current $I$ flows through a coil generating a magnetic field $\mathbf{B}$ in a fluid moving with a velocity $\mathbf{V}$. Let's assume that $\mathbf{B}$ is constant, so that $\partial \mathbf{B} / \partial t=0$ in Eq. (3.26). It remains only the term related to the movement of the conductor in space, and for this simple example the motional electromotive force $(\mathrm{emf})(\varepsilon)$ is as in Eq. (3.25) (slightly modified in Eq. (5.2)).

$$
\varepsilon=V B L
$$

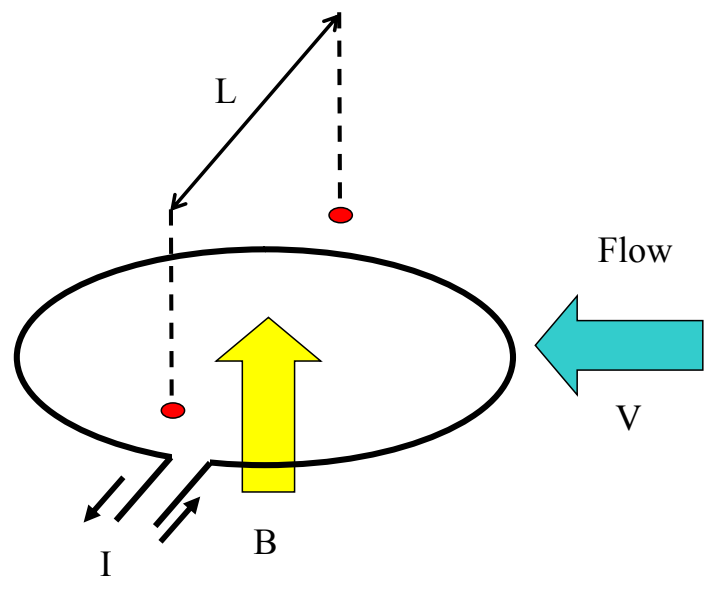

Fig. 5.3: Principle of operation of an electromagnetic flow meter. A current I flows through a coil generating a magnetic field $\mathbf{B}$ in a fluid moving with velocity $\mathbf{V}$. Two electrodes separated a distance $L$ measure the emf $(\varepsilon)$. 
In Figure 5.3 the rod of Figure 3.23 is replaced by a fluid passing through the constant $\mathbf{B}$, and then according to Faraday's law an emf will be induced on the moving fluid. In order to measure the emf two electrodes are placed separated by a distance $L$ in a direction perpendicular to the plane formed by $\mathbf{B}$ and $\mathbf{V}$. The voltage $(v)$ collected by electrodes is proportional to the emf and then to $V$.

Therefore, generating a magnetic field and measuring the voltage on two electrodes the velocity of a fluid could be known. This is the working principle of an electromagnetic flowmeter.

\subsubsection{Current Meters for Environmental Applications}

Figure 5.4 shows a practical scheme of an electromagnetic current meter (EMCM) that measures two components of the currents on the horizontal plane. This kind of instrument is used to know the velocity of water in rivers, lakes and seas. It has two main parts, the coil and the sensing head. The coil is formed by several turns of copper wire wound on a ferromagnetic material which produces the field $\mathbf{B}$ in the water.

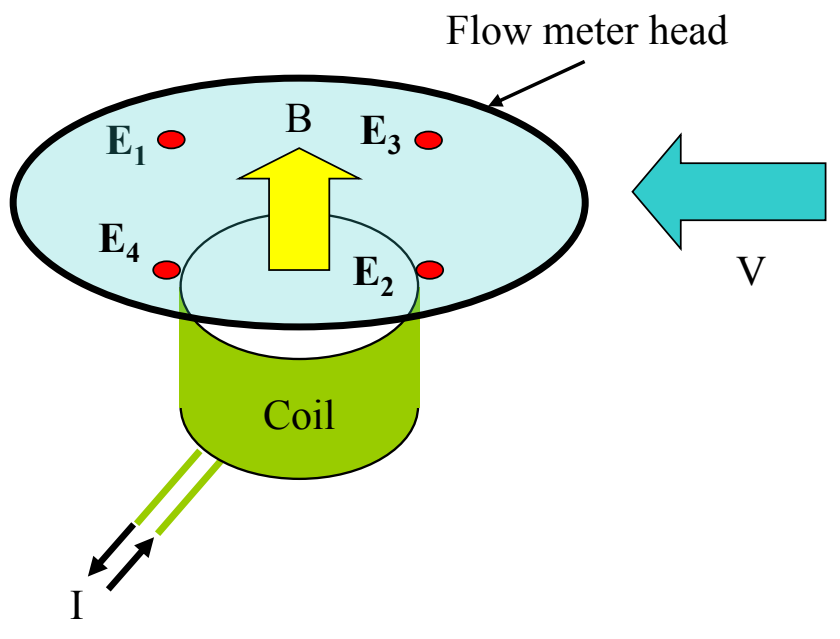

Fig. 5.4: EMCM used to measure the velocity of water on the horizontal plane. The coil generates the magnetic field $B$ on the sensing head. The sensing head has two pairs of electrodes $E_{1}-E_{2}$ and $E_{3}-E_{4}$ arranged in perpendicular directions. They measure two components of the flow velocity based on voltage differences along their respective axes.

The flowmeter head has four electrodes. The pair $E_{1}$ and $E_{2}$ is in a direction perpendicular to that of the pair $\mathrm{E}_{3}$ and $\mathrm{E}_{4}$. Each pair measures a voltage proportional to the emf generated by the flow in its own direction. According to Eq. (5.2) the 
emfs are proportional to the flow velocity, so two components of the velocity along perpendicular axes are measured based on the voltage differences. Usually EMCM have a magnetic compass or a flux-gate compass to refer the velocity to the geographical coordinates giving as a result the north-south and the east-west components of $\mathbf{V}$.

EMCM are available with different sensor shapes. The scheme presented in Figure 5.4 corresponds to the shape known as "discus", which is particularly suitable for laminar flow measurements. There are also "spherical" and "annular" shapes for other flow applications. The spatial resolution of the discus shape is approximately equal to the sensor's diameter, whereas for spherical shape it may be 3 times its diameter. Diameters of commercially available EMCM range from a few centimeters to about $20 \mathrm{~cm}$.

Velocities up to $7.5 \mathrm{~m} / \mathrm{s}$ are measurable with accuracies about $\pm 1 \%$ of the reading. EMCM usually refer their measures to the north-south direction, for this reason manufacturers must specify the compass error or heading error, which may be about $\pm 1^{\circ}$. Some EMCM include a pressure sensor used to measure waves. Wave data combined with current measurements provides wave directional information.

Normally, autonomous current meters have low power consumption and are powered by low voltage cells. Because instruments internally perform some kind of mathematical filtering, each measurement is usually the average of several samples. In most cases the sampling rate and the amount of filtering is user selectable. Another specification that users must be aware of is the depth rating, which usually exceeds the requirements of most common applications.

There are some portable EMCM which are used to take manual readings while wading in shallow water of rivers and streams. In these applications the user should verify that the current meter is able to measure over the full conductivity range, from fresh water to saline water. Sometimes EMCM are mounted on Sea Remote Operated Vehicles to know their speed.

\subsubsection{Industrial Electromagnetic Flowmeter}

Electromagnetic flowmeters (EMFM) for industrial applications have a different design. Generally they are mounted on pipes; Figure 5.5 presents a typical scheme. In this case, the flow has the pipe direction and only one pair of electrodes is enough to know the flow speed. Because industrial environments have a high level of electrical noise, a strong $\mathbf{B}$ has to be produced in order to get a signal that may be recovered from noise. Therefore, two coils adding their magnetic field are employed.

The two coils are interconnected so as to generate an approximately uniform $\mathbf{B}$ across the pipe. Two electrodes are placed inside the pipe in a direction perpendicular to the plane formed by $\mathbf{B}$ and $\mathbf{V}$. They are electrically isolated from the pipe wall and measure the emf induced on the moving fluid so only one of them can be observed 
in Figure 5.5. As before, the voltage measured by the electrodes is proportional to the flow speed.

Electromagnetic flowmeters are the most used in processes involving minerals, food, water, sewage waste and paper pulp. The majority of the aqueous solutions, whether they are clean or dirty, can be measured without any problems. Some important advantages over other flowmeters is that they are easy to maintain, do not produce pressure losses in pipes (because there are no parts protruding inside the tube), and allow flow measurements in both directions.

EMFM are built in diameters ranging from $3 \mathrm{~mm}$ to $2.5 \mathrm{~m}$. The tube used for the construction of the meter must be internally coated with non-conductive material and both the tube and the coating must be non-magnetic. Ceramic and rubber internally covering the tubes allows temperature and chemical resistant instruments to be constructed. Electrodes that are not in direct contact with the fluid have been developed for some applications.

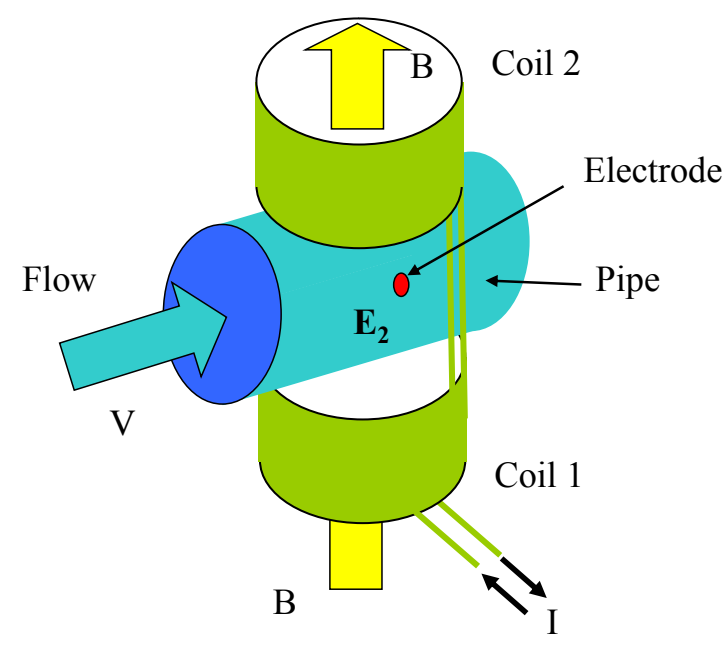

Fig. 5.5: EMFM used to measure the speed of water in pipes. Coils generate a uniform $B$ inside the pipe. One pair of electrodes $E_{1}-E_{2}$ is placed perpendicularly to the pipe direction. It measures a voltage difference proportional to the fluid velocity. Only $\mathrm{E}_{2}$ can be seen in the figure.

The contribution of the fluid velocity to the generation of the emf is greater near the surface of the electrodes and decreases with distance from the electrodes to the center of the tube. This makes flowmeters sensitive to distortion of the speed profile. Some EMFM use four electrodes to be less sensitive to this problem.

Most EMFM measure with errors less than $0.5 \%$ of the measured value for speeds between 1 and $12 \mathrm{~m} / \mathrm{s}$, but for speeds less than $1 \mathrm{~m} / \mathrm{s}$ errors increase significantly, as shown in the Table 5.1, which corresponds to an industrial meter. 
Table 5.1: Error for an EMFM

\begin{tabular}{ll}
\hline $\begin{array}{l}\text { Velocity } \\
(\mathrm{m} / \mathrm{s})\end{array}$ & $\begin{array}{l}\text { Error } \\
\text { (\% of measured value) }\end{array}$ \\
\hline 0.05 & 3 \\
0.1 & 2 \\
0.2 & 1 \\
0.25 & 0.5 \\
1 & 0.3 \\
12 & 0.3 \\
\hline
\end{tabular}

Then, one limitation of this kind of flowmeters is that errors are important for very low velocities. Some EMFM specially designed for low speed flows are able to measure velocities about $0.1 \mathrm{~m} / \mathrm{s}$ with errors of $\pm 1 \%$.

It is important that the conductivity of the fluid be uniform. Otherwise the output signal will be noisy. Also, when measuring liquid which has entrained air, bubbles will cause the EMFM to measure in excess because it assumes that all the fluid is liquid. If the trapped air has the size of the electrode, the output signal could also be noisy.

EMFM must always be full of liquid so the best location for them to be placed is in vertical upward flow lines. Horizontal installation is possible provided that the EMFM is placed at a low point of the pipe line and that the electrodes are not at the top of the pipe (Fig. 5.6). According to manufacturer's recommendation, to keep their accuracy, EMFM require a length of five diameters $(\Phi)$ of straight pipe upstream and two diameters downstream.

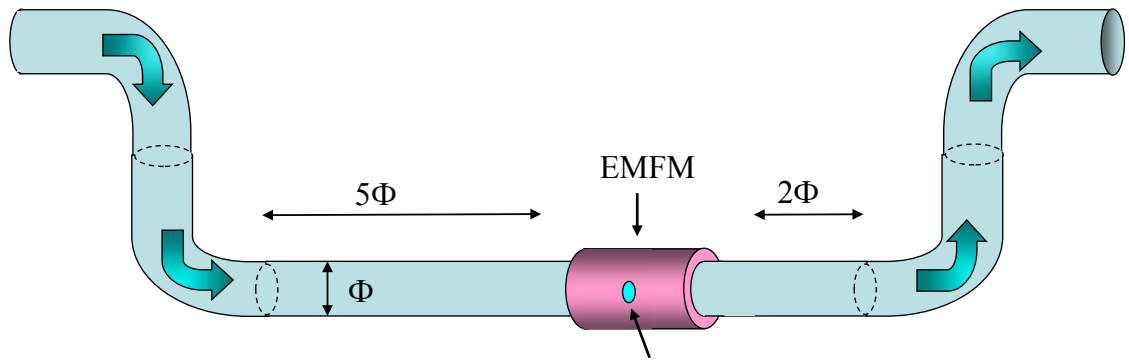

Electrode

Fig. 5.6: When it is not possible to install the flow meter in upward sections of the line it could be installed in horizontal pipes provided that there exists a length of five diameters $(\Phi)$ of straight pipe upstream and two diameters downstream the flow meter. Installation of electrodes at the top or the bottom of the pipe should be avoided in these cases. 
EMFM are also designed as probes to be inserted into pipes through taps. In these designs voltages at the electrodes reflects the velocity at the probe tip and not the average fluid velocity across the pipe. Also, this kind of EMFM produces pressure losses.

\subsubsection{Common Characteristics of EMCM and EMFM}

Until now, to keep the explanations simple, the magnetic field induced in the fluid has been supposed constant, as being produced either by a magnet or by an electromagnet supplied with a DC current. Even when this is theoretically correct, commercial flowmeters do not use a constant $\mathbf{B}$ because chemical instabilities on the electrode surfaces (electrochemical effects) could pollute the signal. The voltages between electrodes due to electrochemical effects are of the order of millivolts. In order to reduce this unwanted effect it is necessary to choose appropriated electrode materials and silver, silver chloride or platinum plated electrodes are commonly used because of their low electrochemical noise.

If a constant B were used, high currents would be necessary in order that electrodes might achieve signal voltages above electrochemical noise. This would lead to coils with impractical sizes and excessive power requirements, a big problem for autonomous instrumentation. Fortunately the electrochemical effects change very slowly with time, and this issue can be addressed by modulating $\mathbf{B}$. Thus, because noise is of low frequency and $\mathbf{B}$ of high frequency, signal can be recovered from noise by frequency filtering. For this purpose manufacturers adopt different waveforms for the current in the coils (Fig. 5.7).

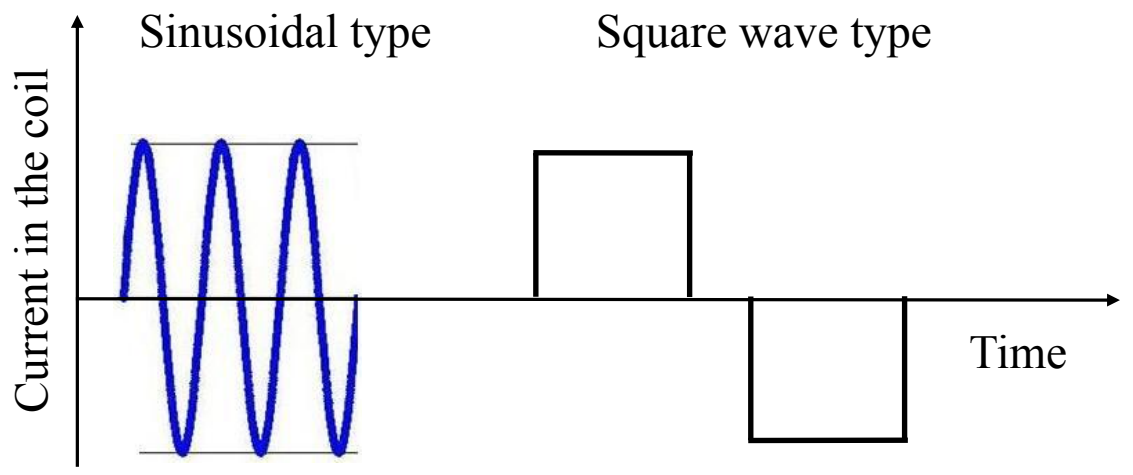

Fig. 5.7: Typical current waveforms in the coils to reduce electrochemical noise effects. 
The generated emf (output signal) is a function of the fluid speed, but is independent of temperature, pressure, density, viscosity, turbulence and, to some extent, of the fluid conductivity. Among the limitations of these flowmeters are that the liquid to be measured must be non-magnetic and should have a minimum conductivity level specified by the manufacturer. The minimum conductivity allowed is generally over $1 \mu \mathrm{S} / \mathrm{cm}$ (drinking tap water conductivity is about $50 \mu \mathrm{S} / \mathrm{cm}$ ).

\subsection{Acoustic Flowmeters}

These flowmeters generate a pressure wave of ultrasonic frequency in the flow being measured. They use piezoelectric narrow beam acoustic transducers (Section (4.13.3)) to send and receive the ultrasound wave. The frequencies employed in commercial meters ranges from 0.5 to $2 \mathrm{MHz}$. Figure 5.8 schematically represents one of the nowadays technologies in which transducers are placed inside the pipe where the flow is measured. The path of the pressure wave forms an angle $\theta$ with the axis of the pipe.

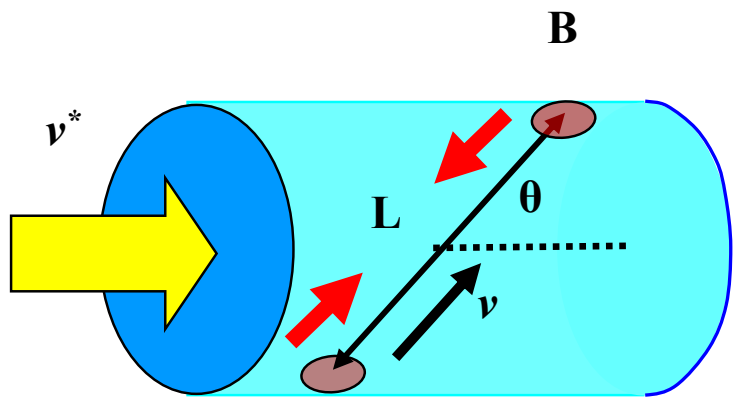

A

Fig. 5.8: Schematic to introduce the basic concepts on acoustic flowmeters. It represents a pipe full of fluid. $A$ and $B$ are acoustic transducers that send and receive ultra sound pulses. The flow $\mathbf{v}^{\star}$ has a component $v$ in the direction of the wave propagation; $\theta$ is the angle between both directions.

The flow through the pipe has a velocity $\mathbf{v}^{\star}$ with a component in the direction of the pressure wave path $v=v^{*} \cos \theta$. If this component has the same direction of the acoustic wave propagation, the speed of the wave will increase. On the other hand, if the acoustic wave has the opposite direction, the speed of the wave will decrease. The fluid velocity can be calculated from the speed of the acoustic wave. For a device such as that depicted in Figure 5.8 there are different ways to measure the wave speed. 


\subsubsection{Transit Time or Time of Flight Method}

\subsubsection{Direct Measure of Time}

This method has some practical limitations but it is useful for introducing the basic concepts on this topic. By means of the transducers an acoustic pulse is sent from A to $\mathrm{B}$, and immediately after, another from $\mathrm{B}$ to $\mathrm{A}$. The transit time $t_{\mathrm{AB}}$ and $t_{\mathrm{BA}}$ are measured and the difference between the times is proportional to the velocity component of the flow in the direction of the wave propagation $(v)$. It also depends on the speed of sound $(c)$ and the distance between sensors $(L)$.

$$
t_{A B}=\frac{L}{c+v} ; t_{B A}=\frac{L}{c-v} ; \quad \Delta t=t_{B A}-t_{A B}=L\left[\frac{1}{c-v}-\frac{1}{c+v}\right]=\frac{2 L v}{c^{2}-v^{2}}
$$

In practice, for most fluids $c^{2} \gg v^{2}$ and $c^{2}-v^{2} \approx c^{2}$ (for example for water $c=1500$ $\mathrm{m} / \mathrm{s}$ and $v=10 \mathrm{~m} / \mathrm{s}$ ). so that Ea. (5.3) becomes

$$
\Delta t=\frac{2 L v}{c^{2}}=\frac{2 L v^{*} \cos \theta}{c^{2}} ; \quad v^{*}=\frac{\Delta t c^{2}}{2 L \cos \theta}
$$

Thus, once the time difference $\Delta t$ is computed, the flow speed $\left(v^{*}\right)$ can be calculated. Because changes in fluid temperature modify the speed of sound in the fluid due to changes in density, this change should be considered when computing $v^{*}$ with Eq. (5.4). The volumetric flow rate could be estimated from $v^{*}$ and the cross-sectional area of the pipe

The time difference $(\Delta t)$ that an instrument should measure for detecting a change in fluid velocity inside a pipe is small. Suppose that the fluid is water ( $c=1500 \mathrm{~m} / \mathrm{s}$ ), $L=0.5 \mathrm{~m}$ and $\theta=45^{\circ}(\cos \theta \approx 0.7)$. If it is desired to detect a speed change of $\Delta v=0.01 \mathrm{~m} / \mathrm{s}$, the time difference to be measured will be:

$$
\Delta t=\frac{2 \times 0.5 \times 0.01 \times 0.7}{1500^{2}} \mathrm{~s} \approx 3 \times 10^{-9} \mathrm{~s}
$$

The simplest way to measure a transit time is to start a digital clock synchronized with the positive slope of the transmitted pulse and to stop it with the positive slope of the arriving pulse. As the acoustic signal arriving to the sensor has noise, the pulse shape is distorted and its slope difficult to detect (Fig. 5.9).

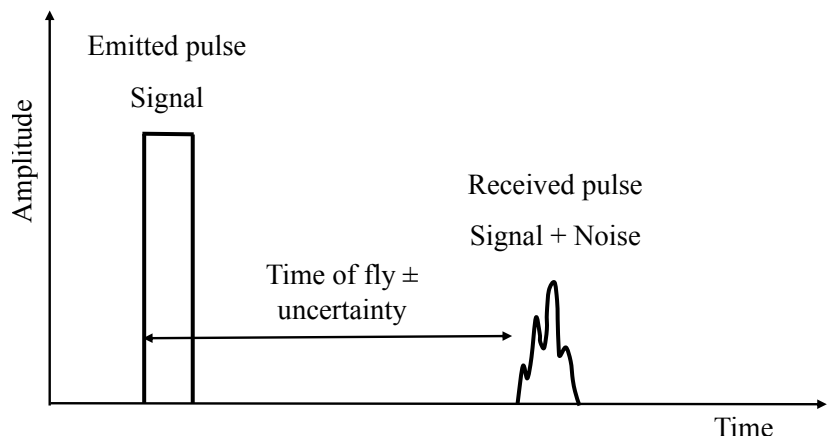

Fig. 5.9: Emitted and received pulses. The received pulse is smaller and noisier; its slope is not well defined, which makes it difficult for the time of flight to be measured. 


\subsubsection{Frequency Difference Measure}

This method uses the same physical scheme of Figure 5.8, but the electronics and the processing algorithm are different. In this case, when a pulse emitted from A arrives to $\mathrm{B}$, another pulse is emitted again from $\mathrm{A}$ to $\mathrm{B}$. This operation is repeated $n$ times. Then the direction of the transmission is reversed, and other $n$ pulses are sent from $\mathrm{B}$ to A. Because $v$ makes the wave velocity increase when traveling from A to B, the first $n$ pulses will be issued in less time than those from $B$ to $A$. The pulse frequency in the first case is greater than in the second. The difference in frequency between both propagation directions $(\Delta f)$ is shown in Eq. (5.5), where $f_{1}$ is the frequency in the flow direction and $f_{2}$ in the opposite direction,

$$
f_{1}=\frac{1}{t_{A B}} ; f_{2}=\frac{1}{t_{B A}} ; \Delta f=f_{1}-f_{2}=\frac{1}{t_{A B}}-\frac{1}{t_{B A}}=\frac{c+v}{L}-\frac{c-v}{L}=\frac{2 v^{*} \cos \theta}{L}=\frac{2 v^{*} D}{L^{2}} \rightarrow v^{*}=\frac{L^{2}}{2 D} \Delta f
$$

The frequency difference is thus proportional to the fluid velocity. Now the speed of sound does not appear in the equation. This is an interesting property of this method since it becomes independent of the temperature and salinity of the fluid and the velocity of the fluid may be obtained from the distance between sensors $(L)$, the pipe diameter $(D)$ and difference in frequency.

The use of a number $n$ of pulses in each direction of transmission reduces the random noise because sometimes it will be added to the signal and sometimes subtracted from it. Thus, on the average, noise will tend to cancel. It should be noted that all deductions were made assuming that $\mathbf{v}^{\star}$ remains the same while both transducers alternate as senders and receivers, i.e. fluid velocity does not change while $2 n$ pulses are sent and received.

\subsubsection{Other Acoustic Flowmeters Characteristics}

Acoustic sensors can be mounted so that the acoustic wave follows a direct path through the fluid as in Figure 5.8, or is reflected on the wall of the tube, reaching the receiver upon rebound as in Figure 5.10. In the second case, the accuracy of the method is increased because the acoustic wave travels a longer distance and "feels more flow". Figure 5.10 shows the constituent parts of a simplified industrial flowmeter.

A preamplifier placed near the transducers allows the junction box, from which the instrument is powered, to be separated from the pipe. The communication port can be used to transfer the flowmeter data to recorders, data acquisition systems, transmission units, etc.

If the mass flow is needed, it is necessary to know the pressure $(P)$ and temperature $(T)$ of the fluid to correct for density changes. For this purpose both sensors are placed downstream to avoid disrupting the fluid regime (Fig. 5.10).

Accuracies of $\pm 1 \%$ are achieved with this type of flowmeters. They are limited by the ability of the signal processing electronics to determine the transit time. Transducers can be placed inside the tube during the manufacturing process or be fixed externally to the walls of the pipes. The last are known as "clamp-on" transducers. Internal 
transducers in contact with the fluid (wet transducers) are usually more accurate, but the externals are easier to install

These flowmeters are able to measure bidirectional flow and the cost is almost independent of the pipe size. Because they are not intrusive, pressure drop (head loss) is practically zero, and because they do not have moving parts they require low maintenance. As in most flowmeters, mounting the transducers in an area where the pipe may become partially filled should be avoided, because partially filled pipes will cause erroneous measurements.

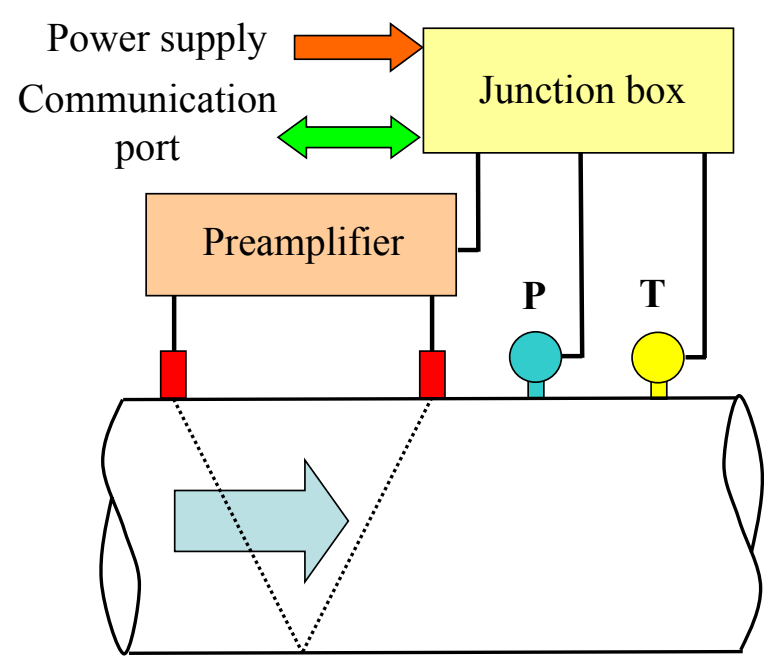

Fig. 5.10: A more complete schematic of a flowmeter shows the pressure $(P)$ and temperature $(T)$ sensors together with the electrical parts. In this flowmeter the acoustic wave rebounds on the opposite wall of the pipe traveling a longer distance.

Instruments with external transducers must be programmed for each pipeline material, diameter, and wall thickness. The pipe must be constructed of an acoustic conducting material. The largest benefit of the external transducers is obviously the ease-of-installation. They are especially useful for short time measurements or when shutting the system down to install the flowmeter is not possible.

The principle of operation of these particular acoustic flowmeters requires that the fluid be clean because if it is not, the sound wave could be reflected/scattered by the fluid particles and would not arrive at the receiver. Also fluid containing air bubbles or eddies, reflects/scatters the sound making the measurements difficult. It is worth noting that the use of terms 'reflected' or 'scattered' depends upon the size of the 'reflecting particle' with respect to incident acoustic wavelength.

If the size of the reflecting particle is larger than the acoustic wavelength (Fig. 5.11b) it is possible to talk about reflection, but if the size of the 'reflecting particle' is 
much smaller than a wavelength the law of reflection can no longer be applied, and one must talk about scattering rather than reflection (Fig. 5.11a). In the first case the reflected waves are basically plane waves, whereas in the second case the scattered waves are essentially spherical waves. Although reflection and scattering are present in most cases, the term scattering will be used from now on for the sake of clarity.

For those meters with "clamp-on" transducers, acoustic discontinuities, as those found in concrete-lined or fiberglass-reinforced pipes, may scatter and attenuate acoustic signals, increasing the flowmeter error to $\pm 20 \%$.

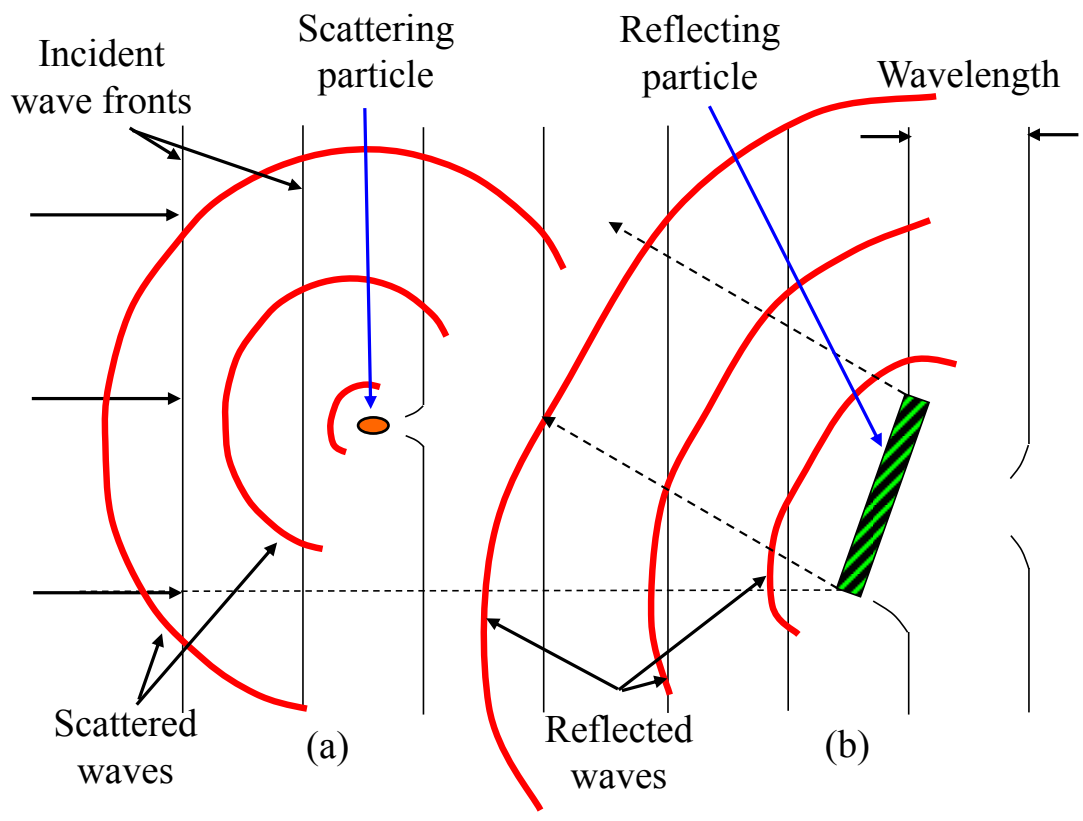

Fig. 5.11: (a) Scattering of sound waves by a particle of size much smaller than a wavelength. (b) Reflection of sound waves by a particle of size comparable to a wavelength.

\subsubsection{Doppler Flowmeters}

Doppler flowmeters (DFM) use piezoelectric transducers to project an ultrasound acoustic beam in the fluid whose speed is to be measured. The beam is emitted at an angle $\theta$ with respect to the direction of the flow and suspended particles or bubbles in the fluid scatter part of the energy received from the acoustic beam (Fig. 5.12). The DFM theory is based on the assumption that particles move at the same speed as the fluid. For this to be true the fluid speed must keep the solids in suspension. 


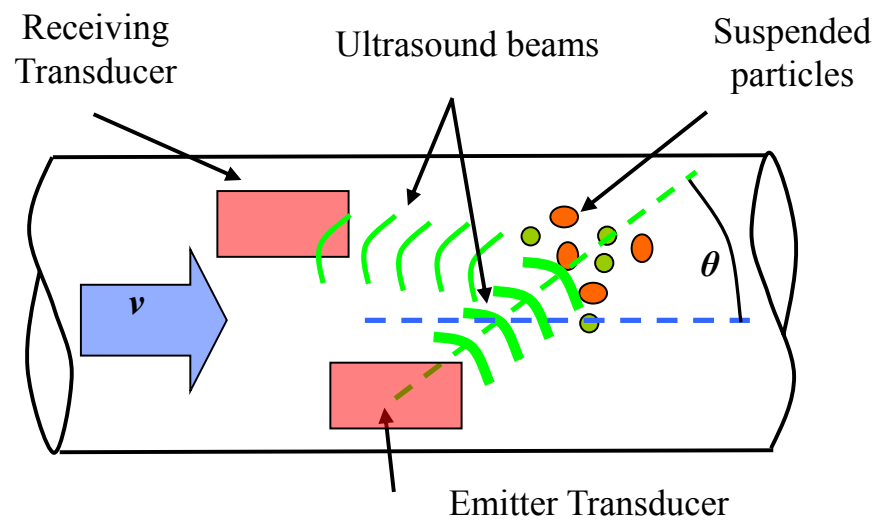

Fig. 5.12: Flow in a pipe. The emitter and receiver transducers are installed such that their beams form an angle $\theta$ with the pipe direction. Emitted pulses are scattered by suspended particles that move at velocity $\mathbf{v}$. The scatters are received by the opposite transducer.

Suspended particles move with respect to the fixed transducers (emitter and receiver) and, according to the Doppler effect (Section (3.3.1)), the frequency at the receiver is given by

$$
\Delta f \approx \frac{2 v \cos \theta f_{0}}{c}
$$

As before $c$ is the speed of sound, $f_{0}$ the emitted frequency, $v$ the flow speed, and $\theta$ the angle between $v$ and the beam direction. The electronic circuitry measures the frequency difference $\Delta f$ and calculates $v$ as

$$
v=\frac{c \Delta f}{2 \cos \theta f_{0}}
$$

Reliable measurements require knowledge of $c$. Because the equation relating temperature to $c$ for different fluids is usually known, measuring the temperature allows correcting $c$ for temperature variations.

\subsubsection{Installation and Characteristics}

Doppler flowmeters should not be mounted on pipes that vibrate excessively. To obtain reliable measurements the installation should be done in sections of the pipe which are always full of fluid, as in vertical upwards flow sections. Sensors should not be mounted at the top and bottom in horizontal sections of the pipe, because fluid's foam (at the top) and deposited sediment (at the bottom) can attenuate the signal excessively.

Weak signals not suitable to obtain reliable measurements may be due to a very clean fluid, low flow speed, pipes with internal coating or large diameter pipes. It is worth noting that Doppler acoustic meters require the presence of particles in the 
fluid, as opposed to the acoustic current meters described above. Then when signals are not strong enough, the addition of bubbles or solids in suspension upstream, whenever possible, would improve the signal to noise ratio.

DFM have low cost of installation since in many cases are simply mounted on the outside of the pipes. There are portable models which are very handy for quick checks of flow in industrial plants. The portability is generally achieved at the expense of accuracy.

Some manufacturers indicate that for adequate signal reflections a minimum of approximately $100 \mathrm{mg}$ per liter of solids with a particle size of about $75 \mu \mathrm{m}$ is required. If solid concentrations are in excess of $45 \%$ by weight the output of the reflected signal could be too low to be correctly processed due to the acoustic wave attenuation.

DFM with external transducers cannot be used in pipes of concrete or plastic reinforced with glass fibers, since the waves could be mostly reflected in the external layers. Pipes with internal protective coatings should be avoided because air could be trapped between the external tube and the internal coating, thus preventing the passage of the signal. DFM work well with PVC tubes, instead.

Although DFM can be used with sewage sludge, highly concentrated sludge precludes wave penetration into the center of the flow. Waves are reflected by the slower fluid near the walls where speeds are lower, which results in measures misleadingly low.

Some manufacturers claim that the lower limit of detectable speed for this technology is between 0.1 and $0.6 \mathrm{~m} / \mathrm{s}$, the accuracy of the measurements being about $\pm 2 \%$ of full scale. An important advantage of DFM is that they produce no pressure drops because they do not interfere with the flow. The most appropriate piping materials where these flowmeters can be used are iron, steel, PVC, plastic and aluminum.

In order to compute the volumetric flow it is necessary to know the internal diameter of the pipe. In old pipes, however, depositions or inlays make it difficult to know the internal diameter. Additionally, inlays can prevent the passage of the acoustic signal precluding measurements, as is the case of calcium in water pipes.

\subsubsection{New Flowmeters (Both Transit Time and Doppler)}

As it has been stressed, transit time flowmeters work in clean water but not in water with bubbles or sediment in suspension. On the contrary, Doppler flowmeters require some material in suspension to reflect the sound wave. Manufacturers realized that the methods have similar sensors and hardware but different data processing. Then they designed an instrument capable of measuring in both cases, fluids containing air bubbles and sediments, and clean fluids. They added some intelligence on board the instruments to detect the degree of cleanness of the fluid. According to the fluid condition the instrument switches automatically to measure using one or the other functioning principle. 


\subsubsection{Acoustic Doppler Current Profiler (ADCP)}

The word "profiler" in the name of this kind of acoustic current meter means that it has the ability to measure flow velocity at different distances from the transducer simultaneously. The distances measured by these instruments may range from some decimeters to several meters.

There are diverse shapes of acoustic current profilers with different amounts of acoustic transducers, but all of them work in a similar way. With the aim of simplifying the functioning description of an ADCP, a profiler which measures two-dimensional (2D) velocities along a channel will be illustrated first. The concepts developed in its description will be used to extend the explanation to three-dimensional (3D) profilers.

\subsubsection{Two-Dimensional Profiler}

This profiler was developed to continuously monitor flow in channels, rivers, pipes, culverts, etc. It has three acoustic beams and is mounted at the bottom of the water course. One of these beams points straight up, and the other two point upstream and downstream at an angle of $45^{\circ}$ (Fig. 5.13). The first beam measures the height of the surface while the inclined beams measure the water velocity using the Doppler shift as explained in Section (3.3.1) and shown in Eqs. (3.8) or (5.7).

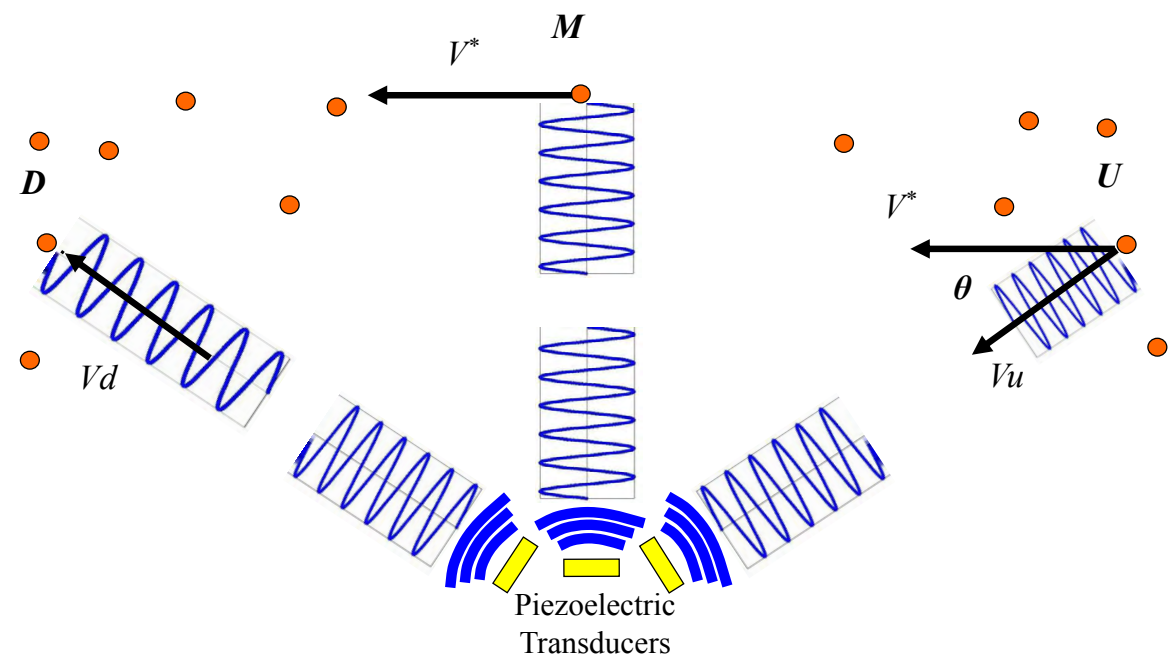

Fig. 5.13: Three piezoelectric transducers emit acoustic waves of the same frequency. Water carries particles in suspension or bubbles at a velocity $\mathbf{V}^{\star}$. Particles $\mathrm{U}$ (upstream) and $\mathrm{D}$ (downstream) produce different frequency shifts; $M$ (in the vertical) scatters the same sent frequency without shift. 
The acoustic transducers used in these meters are piezoelectric ceramics. They are used both for transmission and reception of the acoustic signals, and because of this characteristic the ADCP is called monostatic sonar. Piezoelectric ceramics for these applications produce a narrow beam of sound, so most of the energy is concentrated in a few degrees beamwidth. Sound frequency $\left(f_{0}\right)$ is usually between 1 to $2 \mathrm{MHz}$.

In the first step of the measuring process, the electronics sends an electric pulse to the transducer which generates a pulse of ultrasound. This pulse propagates through the fluid and is scattered in all directions by acoustic heterogeneities present in the water (suspended material, sediment, bubbles, organic matter, etc). The energy scattered by the heterogeneities that the ultrasound wave finds in the direction of the transducer beam is received back by the transducer and converted into an electrical signal. That is to say, the transducer works, firstly converting electrical pulse energy into vibration in the fluid, and secondly receiving vibrations coming back from suspended particles and converting them into an electric signal. The frequency of the received back signal $(f)$ is compared to that emitted $\left(f_{0}\right)$ and the Doppler shift is calculated $\Delta f=f-f_{0}$ by the on board electronics, thus the velocity of the fluid is calculated from Eq. (5.7) if $c$ is known.

The three piezoelectric transducers are schematically represented in Figure 5.13. They emit acoustic waves of the same frequency. The dots represent particles or bubbles in suspension. Water is supposed to move at a velocity $\mathbf{V}^{\star}$. Particle $\mathrm{U}$ (upstream) has a velocity component $V u$ along the direction of the right transducer beamwidth. Particle D (downstream) has a velocity component $V d$ along the direction of the left transducer beamwidth. Both $V u$ and $V d$ produce frequency shifts. Particle $\mathrm{U}$ scatters sound at a higher frequency meanwhile particle D scatters it at a lower one. Particle M (in the vertical) has no velocity component in the direction of the vertical beamwidth, so it scatters the same sent frequency without shift. The maximum reflection received back at the vertical transducer is produced by the water surface.

For known sound speed propagation, the elapsed time since the pulse was emitted and received back allows the distance between the piezoelectric transducer and the scattering particles to be calculated. Therefore, by calculating the Doppler shift for each distance, the velocity profile of the particles at different distances from the transducer can be estimated from the beams pointing upstream and downstream. The central beam with no frequency shift is used to measure the distance from the instrument to the water surface.

In order to process and record velocity information as a function of distance, the transducer's beam has to be discretized at regular intervals. The spatial averaged velocity of the particles on these intervals is estimated. Then, the transducer beam is divided into equal length fractions called cells (Fig. 5.14). Generally, users can select the total number and the length of the cells from a set offered by the manufacturer. Because transducers need a certain time interval to switch from emission to reception, no velocity can be measured in the portion of the beam closest to the transducer. This portion is called the blanking distance. 
This blanking distance and the distance to the farthest cell measured from the transducer's surface, depend on the transducer's characteristics and the pulse length. For a real instrument they can be on the order of $0.1 \mathrm{~m}$ and $5 \mathrm{~m}$, respectively. The beams should be free from any obstruction, at least for the total measuring distance.

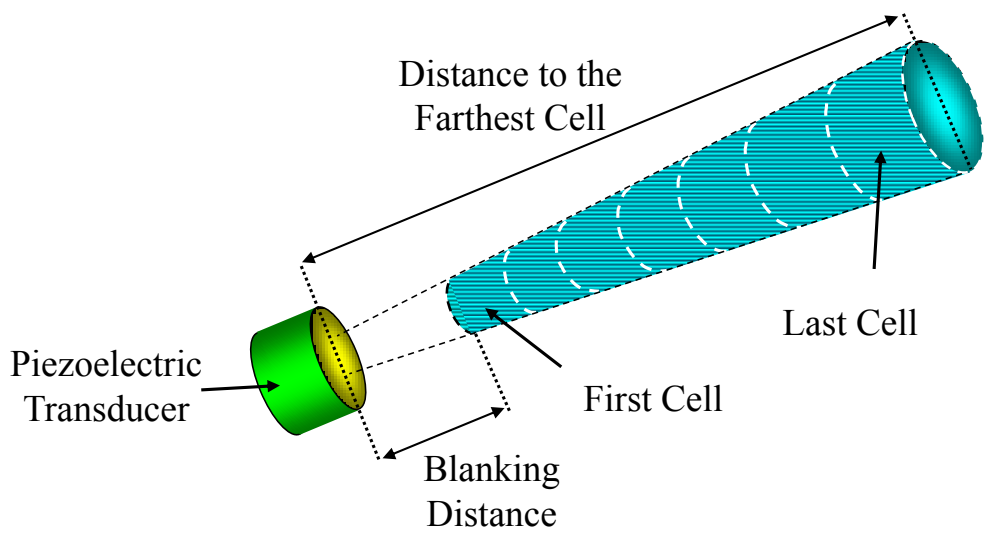

Fig. 5.14: The transducer beam of the piezoelectric transducer is divided into equal length fractions called cells. Average velocities in each cell are measured. No velocity can be measured in the portion of the beam called the blanking distance.

This kind of ADCP must be mounted such that the plane containing the transducer's beams is aligned with the stream. A schematic of an ADCP installation in a natural channel is illustrated in Figure 5.15.

\section{Acoustic beams}

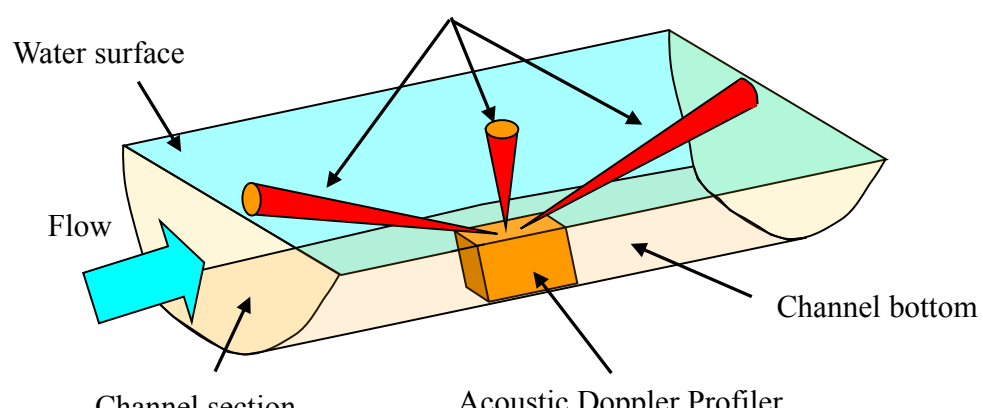

Channel section

Acoustic Doppler Profiler

Fig. 5.15: The ADCP is installed at the bottom of a natural channel. The plane containing the three transducer's beams is aligned with the stream. 
From the Doppler shifts both beam velocities $V u$ and $V d$ are calculated and converted into the along-channel velocity and vertical velocity through the beam geometry. Under channel or river conditions, the along-channel velocity is the main velocity, the vertical velocity being quite small.

Figure 5.16 shows the installation of an ADCP in a channel where it is expected to find sediment deposits. The stream velocity direction is indicated by the arrow and the three discs (yellow) are the acoustic transducers. Sediment on transducers attenuates the sound wave and hinders measurements to be performed correctly. To avoid sediment deposition on the transducers the profiler was separated from the channel floor. This separation from the bottom has to be considered in flow calculations.

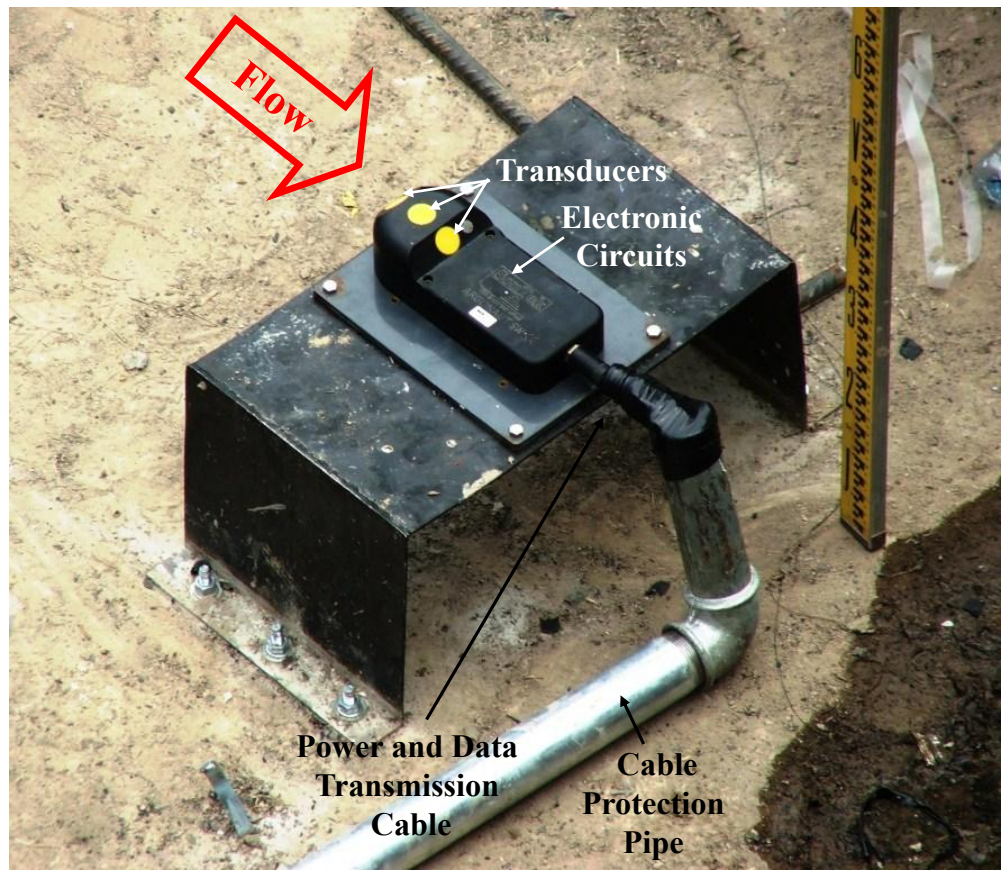

Fig. 5.16: Photography of a two-dimensional profiler installed at the bottom of a concrete channel. The discs are acoustic transducers mounted such the stream velocity is aligned with the plane containing the transducers. Protection to the cable is provided by a pipe. A metal U-shaped mounting device, bolt to the floor, is used to avoid sediment deposition on the transducers.

In this kind of ADCP that measures the water height, the cross-sectional area can be calculated by the instrument if the geometry of the channel is known. Then using the velocity profile data, the volumetric flow rate can be estimated. For this ADCP the manufacturers claim an accuracy of $\pm 1 \%$ of the velocity measuring range $(5 \mathrm{~m} / \mathrm{s})$. 
When the flow to be measured carries solids that could damage the ADCP (such as stones in mountain river or solid waste in a discharge channel of a city), the installation at the bottom has to be avoided. Some manufacturers offer instruments to be mounted in a vertical wall of a channel or on a pile in a river. These ADCP measure the velocity profile in a horizontal plane with two beams and the water height with the third beam (Fig. 5.17).

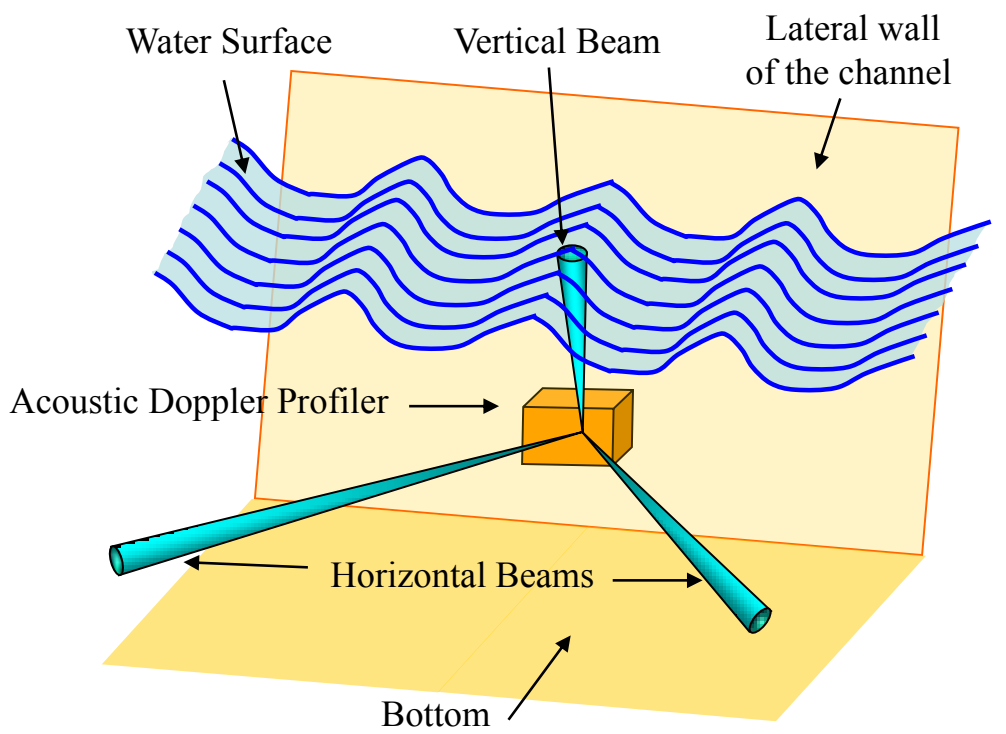

Fig. 5.17: ADCP mounted on the lateral wall of a channel, it measures the horizontal profile with two beams. The third beam points upward to measure the water height. This arrangement could be used when the flow to be measured carries solids that could damage the instrument if mounted on the bottom.

\subsubsection{Three-Dimensional Profiler}

Although, as noted above, there are ADCP with different geometries, all of them can be understood as an extension of the previous 2D profiler because they work on the same functioning principle.

In order to capture 3D information, for instance in open seas, the first idea that comes to mind is to install two 2D ADCP with their vertical measuring planes perpendicular to each other; for example, installing one with its measuring plane in the north-south direction and the other in the east-west direction. Then, combining the information gathered by the two ADCP a 3D current map can be drawn. 
In principle, two 2D ADCP as that shown in Figure 5.16 could be combined in one instrument with five transducers, one pointing straight up, and the other four on two orthogonal planes and pointing up with their beams at a certain angle from the vertical (Fig. 5.18).

The first beam measures the height of the surface while the inclined beams measure the water velocity profiles on two perpendicular planes. As previously described, the energy scattered by the suspended particles along the direction of each beam is converted back to an electrical signal, and the Doppler shift calculated in the same way as before.

Profilers similar to this description are used to measure in open waters and they are mounted at the bottom or on a moored subsurface buoy. They may be deployed in $40 \mathrm{~m}-180 \mathrm{~m}$ of water and can measure directional waves and currents. They use a magnetic compass and a tilt sensor to refer measures on the planes containing the transducers' beams to the north-south and east-west planes. There are some models of ADCP with arrangements of 3 and 4 sensors which do not require the vertical transducer to obtain the same information (Terray et al., 1999).

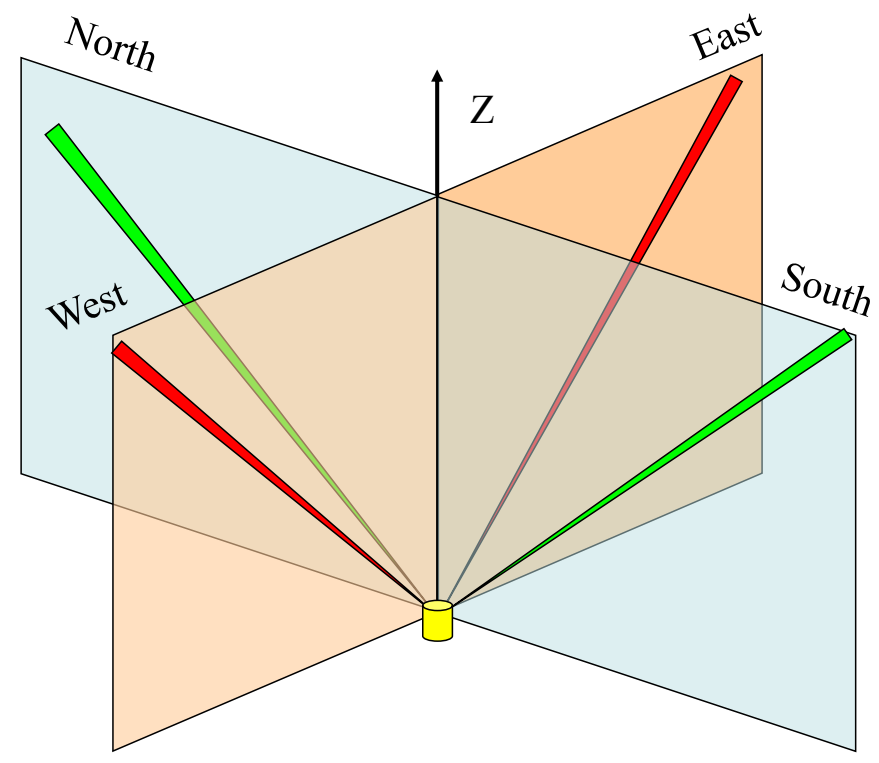

Fig. 5.18: Three-dimensional ADCP, four transducers are place on two orthogonal planes pointing up with their beams at a certain angle from the vertical. They measure the water velocity profiles on two perpendicular planes.

Similar arrangements to those previously described can be mounted looking down on a boat or a buoy for surveying flow in rivers or lakes. 


\subsubsection{ADCP General Characteristics}

With the purpose of having reliable results, some measuring conditions that could limit the utilization of ADCP will be mentioned below and potential users of this technology should verify that their applications are not under the influence of these circumstances.

For a low number of acoustic heterogeneities (low number of particles) in water the energy backscattered to the transducers will be low and the electrical signal weak. Also, for a given number of particles the magnitude of the received backscattered signal decreases with their distances to the transducers. The farthest particles will produce the weakest signals. If the signal strength approaches the noise level, the measured velocity could be unreliable. Usually, low signal to noise ratio indicates less reliable data.

Some manufacturers record the signal to noise ratio so that users may judge the quality of the data. With this information, users could decide if the recorded velocity should be kept or discarded. For each application at hand, users should define the minimum amount of signal to noise ratio that they will accept to consider the data as valid. In order to define this figure, users should perform some previous tests in a tank where measuring conditions are controlled and known velocities could be generated.

In order to correctly measure distances from the transducers to the heterogeneities, the instrument needs to know the actual sound propagation speed in the fluid, which depends on temperature and salinity. ADCP should measure both parameters for sound speed corrections but, in general, they measure only temperature, which has the greatest influence on the speed of sound. Usually users are asked by the instruments to introduce the expected water conductivity at the beginning of the record. This value is used in the internal speed of sound calculations so that to have reliable distances users should have a good estimation of water conductivity. The conductivity should remain constant during the record, or its value changed when appropriate.

Because this method measures the velocity of particles and indirectly estimates that of the water, the assumption that the particles match water velocity has to be accomplished to measure water velocity correctly.

\subsubsection{Acoustic Doppler Velocimeters}

Acoustic Doppler Velocimeters (ADV) also use Doppler frequency shift produced by particles moving in the water to measure flow velocity, but instead of measuring a profile they give a single measure as representative of a given volume. Generally, these volumes are small, which permits a high spatial resolution to be offered.

Figure 5.19 presents an ADV that measures 3D water velocity in a small volume of fluid. Because of this it is useful to measure velocity in laboratory models. The 
measuring principle and the technology employed by this instrument have some points in common with Doppler Flowmeters and Acoustic Doppler Current Profilers, but some important differences will be noted.

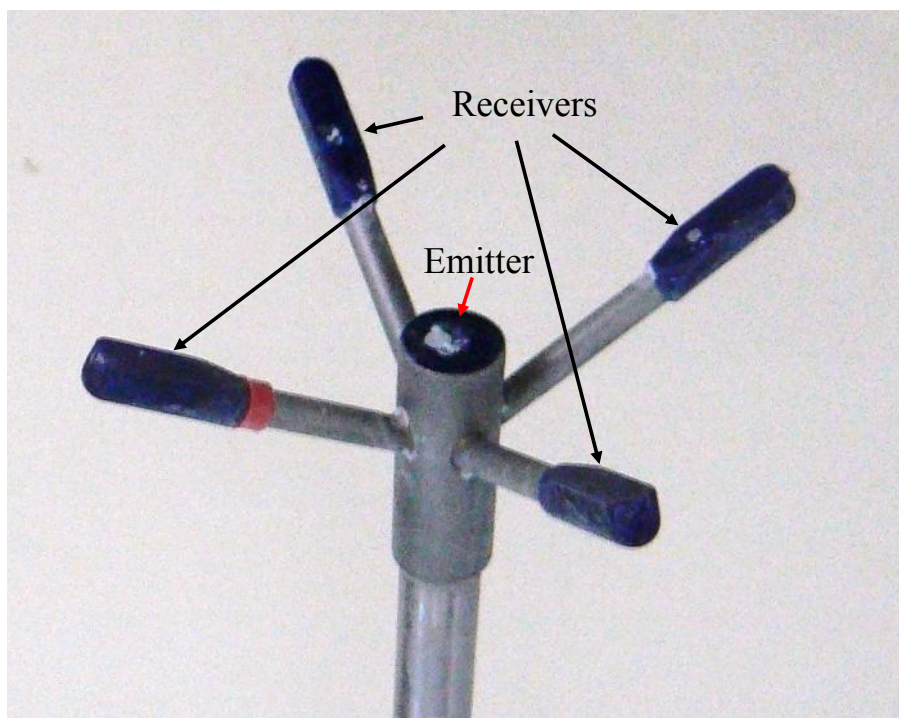

Fig. 5.19: Photography of an Acoustic Doppler Velocimeter. It shows the central emitter and the four receivers forming a cross around the emitter.

To calculate fluid velocity an ADV takes the information from a sample volume of the fluid quite close to the transducers. The distance of the sample to the transducers may range from 50 to $200 \mathrm{~mm}$, depending on the manufacturer. Then an ADV does not measure a profile, but measures in a specific spot of fluid.

ADCP were called monostatic sonars because their transducers are used for both sending and receiving sound waves. Instead, an ADV uses one transducer to send acoustic pulses and three (or four, depending on the model) to receive the scattered acoustic signals from suspended particles passing by the specific spot (Fig. 5.20). Because of this particular characteristic of the transducers ADV are called bistatic sonars. This arrangement of the transducers is sensitive to the velocity component along the direction of the angular bisector between the transmitter and receiver beams. Particles moving at the velocity $\mathbf{V}$ have components $V \mathbf{u}$ and $V \mathrm{~d}$ along the angular bisector directions. For this reason, ADV are more sensitive to the velocity component parallel to the transmitter beam, yielding lower measurement uncertainties along this direction.

For the sake of clarity, only the central emitter and two receivers are shown in Figure 5.20. The emitter has a narrow beamwidth (shown vertical in Figure 5.20) that 
is intersected by the receivers' beamwidth and this spatial intersection defines a "sampling volume". In other words, each receiver's beamwidth is pointed to the same particular volume, and then it senses the acoustic signal scattered by the particles in a specific region of the emitting beamwidth.

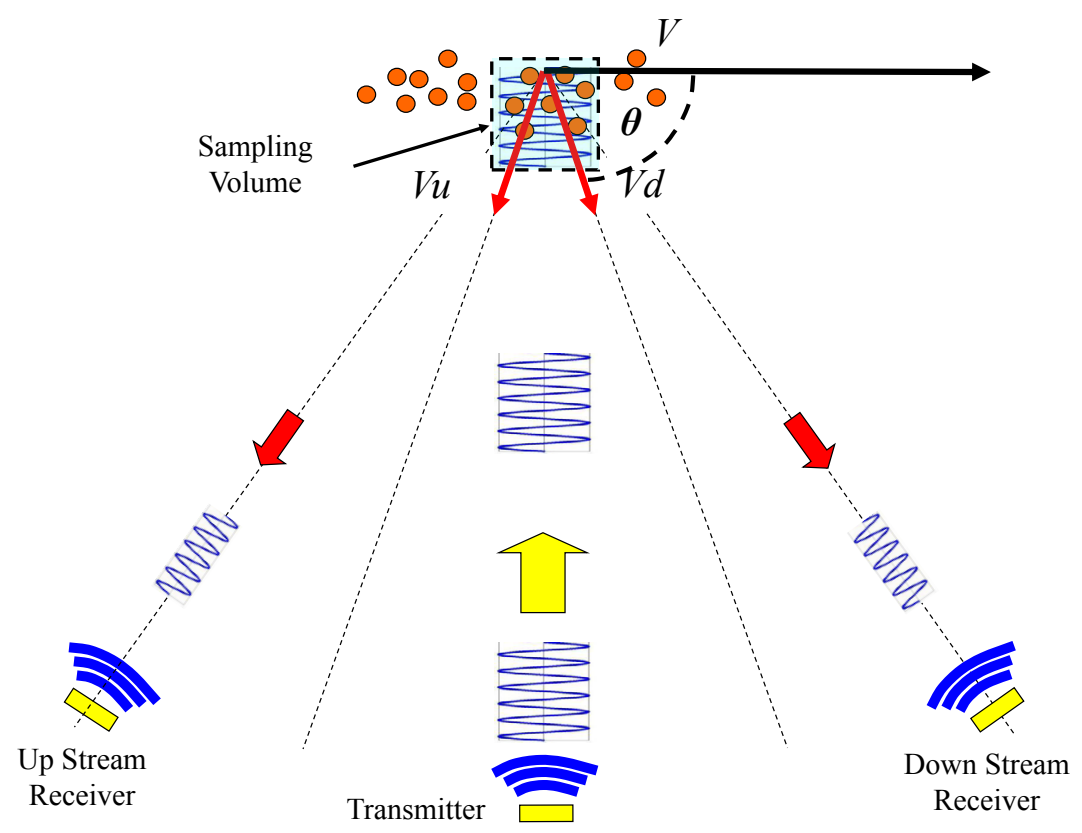

Fig. 5.20: The central emitter and only two receivers of the ADV are schematized. The emitter has a vertical beam that is intersected by the receivers' beams; this spatial intersection defines a "sampling volume". Acoustic signal scattered by the particles in that specific volume arrive at the receivers. The emitter sends short pulses, and the receivers open a sampling time windows to catch the emitted pulse scattered in that volume.

The emitting transducer sends short acoustic pulses, and based on the knowledge of the speed of sound in the fluid, the electronic circuits calculate the time required by the pulse to travel from the emitter to the sampling volume and back to the receivers. A synchronized time window is opened by the electronics to listen and capture only the echo caused by the heterogeneities found in the sampling volume. This technique improves the signal to noise relation.

Water salinity and temperature values are required to permit corrections of sound velocity propagation in water. Usually the first is manually introduced by users and remains fixed while temperature is continuously measured by an on board sensor and used for automatic correction of sound speed. 
The spatial resolution of the measurements depends on the beamwidth and frequency of the transducers. With the aim of achieving the above-mentioned high spatial resolution, the transducer's frequency is about $10-16 \mathrm{MHz}$, approximately one decade higher than the frequencies used by the ADCP.

Each emitted pulse covers a length of a few millimeters. For example, for one commercial ADV each pulse covers between 3 and $15 \mathrm{~mm}$ (users adjustable), which for a $10 \mathrm{MHz}$ frequency in water $(c=1500 \mathrm{~m} / \mathrm{s})$ it is about from 20 to 100 periods of the emitted wave. The higher the amount of periods sent (and received) the lower the uncertainties in the Doppler shift calculation because more signal is processed, which improves the signal to noise ratio.

For a given concentration of moving particles, if it were desired to increase the spatial resolution the emitted pulse should be shortened, and so the amount of emitted wave periods decreases. In so doing, the amount of signal received back to be processed decreases, and since the noise is constant, the signal to noise ratio decreases. Eventually the reliability of the calculated velocity decreases.

When a high spatial resolution is required and the signal to noise ratio is so low that the reliability of the calculated velocity is not acceptable a possible solution would be to artificially increase the particle concentration by seeding some particles (for example, adding some insoluble powder to the water such as talc). Because the energy that arrives to the receivers depends on the concentration of scattering particles, sometimes seeding the water with scattering material provide adequate return signal strength. This improves the signal to noise ratio (SNR) resulting in more accurate velocity measurements. Unfortunately, seeding the flow is not always desirable or practical. SNR is a mean of evaluating the quality of the velocimeters measurements and, in general, manufacturers provide this figure, which advice the user about the quality of the data being acquired.

Figure 5.21a shows the velocity of a turbulent flow measured in a laboratory channel downstream of a hydraulic jump where the particle concentration has been increased. It can be observed that the velocity varies around a mean value of $0.3 \mathrm{~m} / \mathrm{s}$.

Figure 5.21b shows a noisy record from a similar experimental set up. Mean velocity is around $2 \mathrm{~m} / \mathrm{s}$ but spikes of about $\pm 2 \mathrm{~m} / \mathrm{s}$ due to low particle concentration are observed. If only the average velocity is needed spikes could be filtered, but when high frequency hydraulic phenomena are analyzed spikes have to be avoided.

The emitted pulse which contains several periods of the emitted wave is usually referred to as a ping; each ping permits the water velocity in the sampling volume to be estimated. Many pings are averaged together to reduce measure uncertainty giving as a result just one data. The standard data collection rate is about 25 data per second, but some ADV models collect 50 and 200 data per second.

Because the instrument is more sensitive to the velocity component parallel to the transmitter beam, it should be ideally mounted with the transmitter beam parallel to the expected mean velocity of the fluid; therefore, it would yield lower measurement 
uncertainties. But, if installed in this way, the instrument itself would disturb the flow being measured because transducers would stand in the flow way.
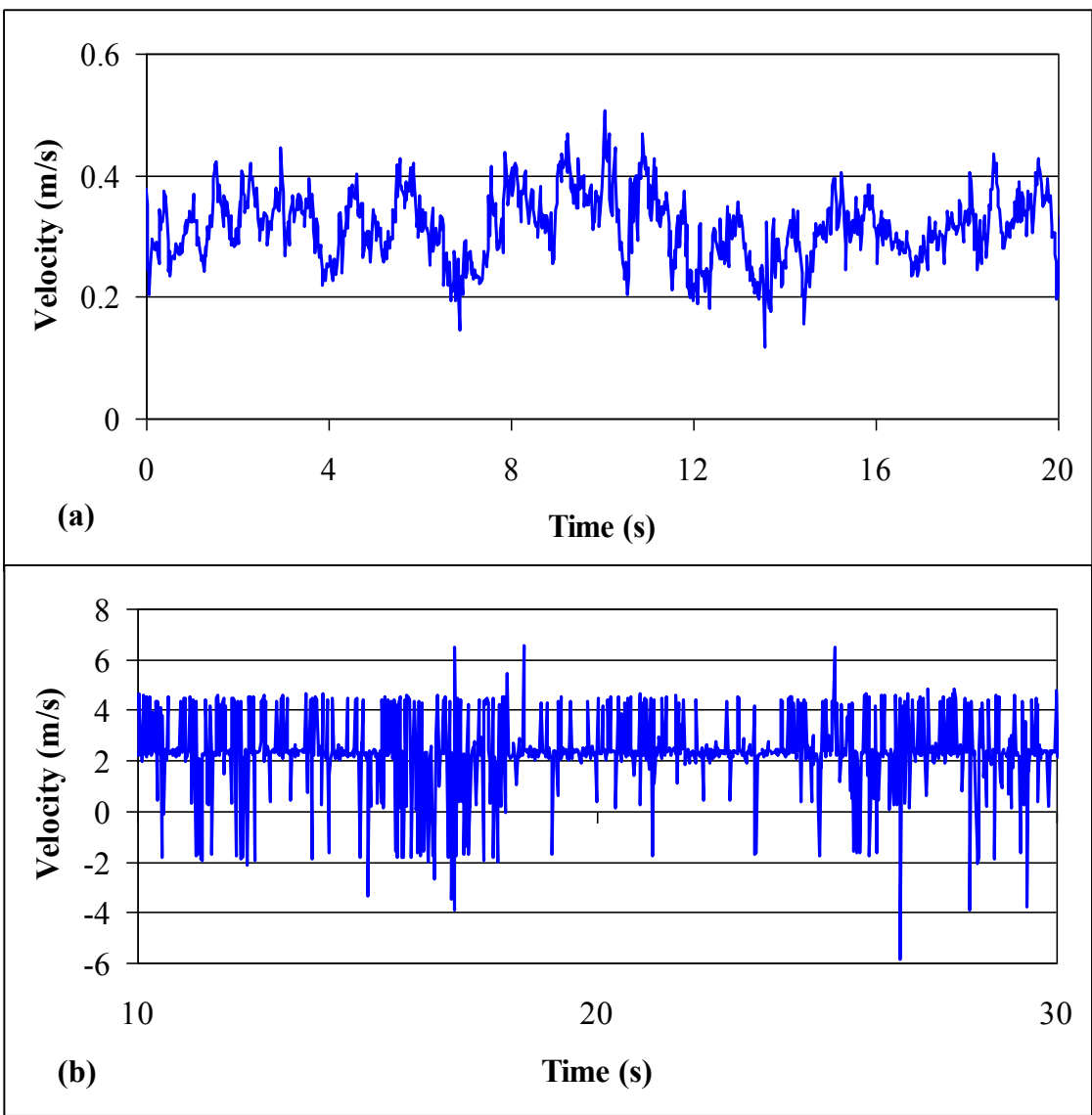

Fig. 5.21: Records of velocity measured with an ADV. (a) Increasing particle concentration improves data quality. (b) Low particle concentration produces noisy records. (Courtesy of Juan Parravicini and Mariano De Dios, Laboratory of Hydromechanics, Hydraulics Department, Faculty of Engineering, National University of La Plata, Argentina).

From the hydraulic point of view, the minimal flow disturb (best data quality) is achieved when flow direction is perpendicular to the transmitter axis because flow perturbation due to the instrument itself is minimized.

By virtue of the foregoing, there is a compromise in the way the instrument should be mounted with respect to the main flow direction because the direction of maximum sensitivity is that of the maximum hydraulic perturbation. The manufacturer advises 
avoiding flow measurements into the transmitter axis to prevent flow disturbance, but when the concentration of particles in water is low, flow perpendicular to the transmitter axis could result in low sensitivity and high noise.

\subsection{Flow Measurements Based on Heat Transference}

Two kinds of thermal mass flowmeters will be described below. One of them supplies a constant heat to the moving fluid and measures a differential temperature which, under some conditions, is proportional to the mass flow. The other computes the amount of electrical power required to maintain the temperature of a heater exposed to the flow stream. Here, the injected power is related to the mass flow.

There is another instrument based on heat transference, called hot wire anemometer, which is used for high flow speed measurements in which the electrical power to keep sensor's temperature constant is representative of fluid velocity.

Although the theoretical models expressing the thermodynamics operating principle for each type of instrument have some equations in common, these equations are, however, somewhat different and they will be developed only in a simplified and conceptual way.

\subsubsection{Introduction to Heat Transfer}

A general explanation of some of the heat transference processes used in these instruments is presented below with the aim to help the reader understand how these meters work.

When the temperature of a body changes it is said that the body has received or given a certain amount of heat. Heat is energy, and although sometimes expressed in calories (cal), its SI unit is the Joule (J) $(1 \mathrm{cal}=4.186 \mathrm{~J})$. The amount of heat required to heat a body is proportional to its mass.

The amount of heat $Q(\mathrm{~J})$ necessary to increase the temperature of a mass $m(\mathrm{~kg})$ from $T_{1}$ to $T_{2}\left({ }^{\circ} \mathrm{C}\right)$ is given by

$$
Q=m c\left(T_{2}-\mathrm{T}_{1}\right)
$$

where $c$ the specific heat of the body $\left(\mathrm{J} \mathrm{kg}^{-1}{ }^{\circ} \mathrm{C}^{-1}\right)$. Equation (5.8) is valid for a certain range of temperature, since for large temperature variations the specific heat capacity is not constant.

Equation (5.9) is the equation for convection of heat (natural and forced) through a surface of area $A$.

$$
q=h_{c} A\left(T_{2}-T_{1}\right)
$$


where $q$ is heat transferred per unit time, $h_{c}$ is the convective heat transfer coefficient, $T_{2}$ is the temperature of the surface and $T_{1}$ the temperature of the fluid. The convective heat transfer coefficient depends on the type of media (gas or liquid) and the flow properties such as velocity and viscosity, among others.

The amount of heat dissipated during a time interval $\mathrm{D} t$ in an electrical resistance $R$ connected to a voltage source $E$ and through which an electric current $I$ flows is given by

$$
Q=R I^{2} \Delta t=\frac{E^{2}}{R} \Delta t
$$

If the resistance $R$ is placed into a fluid, the energy dissipated in the resistance may be used to heat the fluid, and the fluid temperature rises from $T_{1}$ to $T_{2}$ according to Eq. (5.8). Then, if the fluid is moved, it will transport heat from the resistance as expressed by Eq. (5.9). These three simple equations are combined to explain the working principle of the above mentioned instruments.

Units used in the above equations are presented in Table 5.2.

Table 5.2: Units of heat transfer equations

\begin{tabular}{lllllllll}
\hline $\mathbf{m}$ & $\mathbf{I}$ & $\mathbf{E}$ & $\mathbf{T}$ & $\mathbf{R}$ & $\mathbf{Q}$ & $\mathbf{A}$ & $\mathbf{c}$ & $\mathbf{h}_{\mathbf{c}}$ \\
\hline $\mathrm{kg}$ & $\mathrm{A}$ & $\mathrm{V}$ & ${ }^{\circ} \mathrm{C}$ & $\Omega$ & $\mathrm{J}$ & $\mathrm{m}^{2}$ & $\mathrm{~J} \mathrm{~kg}^{-1}{ }^{\circ} \mathrm{C}^{-1}$ & $\mathrm{~W} \mathrm{~m}^{-2} \mathrm{o}^{-1}$ \\
\hline
\end{tabular}

\subsubsection{Mass flowmeters}

There are two types of thermal technologies that measure fluid mass flow directly. They are based on the principles of heat transfer. Both have a heated surface that transfers heat to the molecules of the flowing fluid (McMahon \& Rouse, 2008). Because the types of thermal mass flowmeters have different physical implementations, their respective theoretical thermodynamic models are not the same. In one of the methods the thermal energy is used to heat the fluid, whereas in the other the energy heats a cylinder immersed in the fluid. In order to present the functioning principles simplified models will be adopted.

\subsubsection{Measuring Temperature Difference}

These flowmeters are based on the heat transfer between a hot body and the fluid that passes by and cools it. An outline of the principle is seen in Figure 5.22, where flow in a pipe is being measured. The fluid is heated at the midpoint between two temperature sensors. When the fluid is at rest heat spreads symmetrically and $T_{1}=T_{2}$. 
When a fluid begins to move, as indicated in the figure, a certain amount of heat is transported from point 1 to point 2 , so that $T_{1}$ decreases and $T_{2}$ increases with respect to the previous situation. Within a particular range of flow speeds, and for certain fluids, the temperature difference is proportional to the mass per unit time flowing through the pipe,

$$
\frac{\Delta m}{\Delta t}=K\left(T_{2}-T_{1}\right)
$$

If the speed of the fluid increases beyond a certain point the relation between mass flow and temperature difference is no longer linear.

Equation (5.11) shows that injecting heat by means of a heater and measuring temperature upstream and downstream the injection point, the mass flow may be estimated. The constant $K$ is the slope of the flowmeter transfer and can be obtained through a calibration process.

\section{Heater}

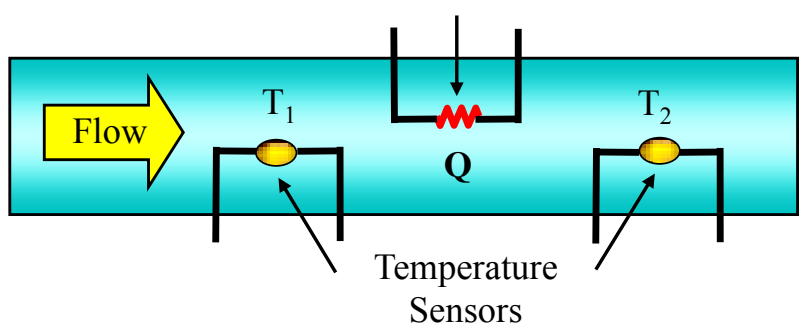

Fig. 5.22: Flow through a tube is heated at the midpoint between two temperature sensors ( $T_{1}$ and $T_{2}$ ). Fluid transports heat from point 1 to point 2 .

There are other designs based on the same principle (Fig. 5.23) where two heated temperature sensors are used. Sometimes they simply consist of two platinum resistance temperature detectors (RTD) (Section (4.7.2)). They are identical and equally heated by means of an electronic circuit so that with null flow both sensors will be at the same temperature. When fluid moves as in the figure heat is transported by the fluid mass, resulting $T_{2}>T_{1}$ and Eq. (5.11) is met as in the previous design.

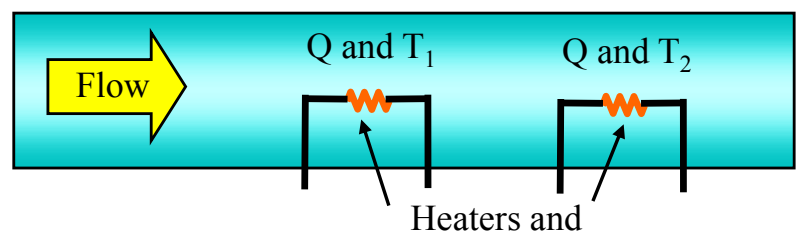

Sensors

Fig. 5.23: Two heated temperature sensors are used to heat the fluid and to measure its temperature at two points. Sensors may be resistance temperature detectors (RTD). 
The measurement schemes presented in Figures 5.22 and 5.23 are used for small pipe diameters and small flow rates. Otherwise, the amount of heat that must be provided to generate measurable differences in temperature would be very great. With the purpose of using this technique in larger pipe diameters its practical implementation has to be modified as in Figure 5.24.

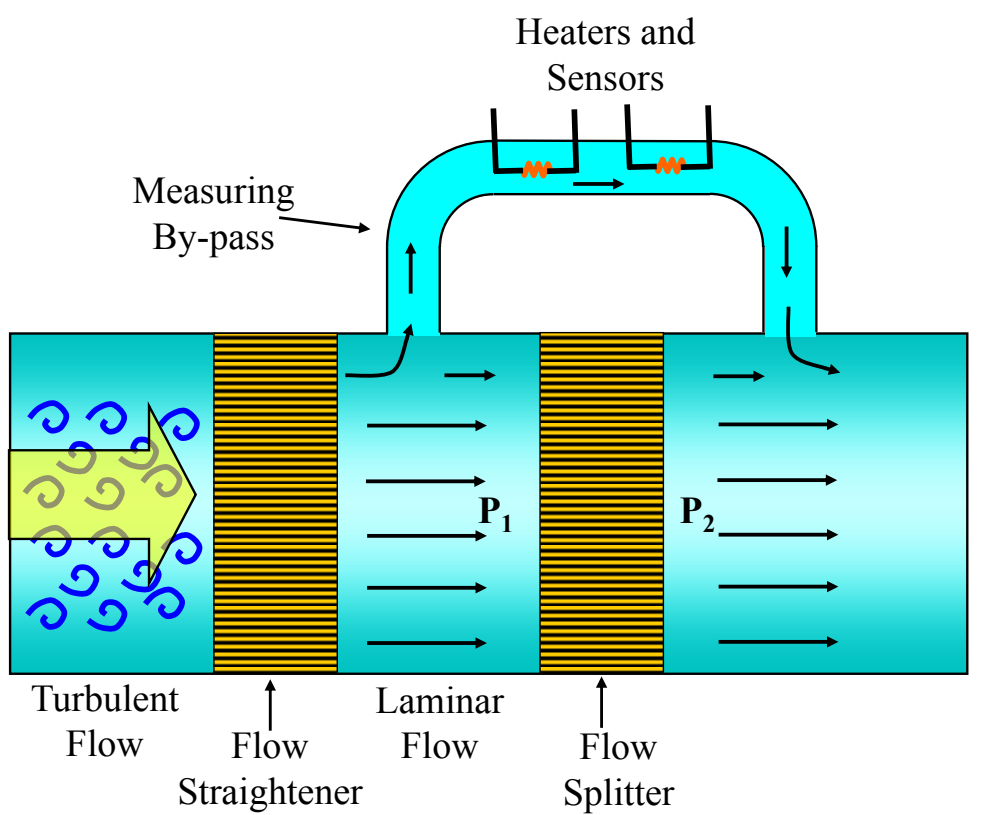

Fig. 5.24: The technique is modified to be used in larger pipe diameters. The flow straightener converts turbulent flow into a laminar one. A flow splitter produces a differential pressure $\left(\Delta P=P_{1}-P_{2}\right)$ which derives the fluid into the by-pass where the measuring system is installed. Flow through the by-pass is proportional to that passing through the main tube.

Figure 5.24 shows that by means of a flow straightener the turbulent flow at the input is converted into a laminar one. Downstream a measuring by-pass, a hydrodynamic resistance (flow splitter) is introduced to produce a differential pressure $\left(\triangle P=P_{1}-P_{2}\right)$ which derives the fluid into the by-pass (http://sierratechsupport.com). The amount of fluid that goes by the measuring system (heaters and sensors) is proportional to that passing through the main tube. Therefore, measuring the differential temperature in the smaller tube allows the total mass flow through the pipe to be calculated.

This technology requires simple electronic circuits, since they basically need measuring temperature differences. This type of flowmeter is suitable for measuring in air, nitrogen, hydrogen, oxygen, helium, ammonia, argon, carbon monoxide, carbon dioxide, hydrochloric acid, ethane, ethylene, methane, etc. These flowmeters 
are not useful to measure in fluids whose $c$ (specific heat) varies with temperature or time (such as could occur in mixtures). It is a very useful technique for detecting slow fluid movements such as leaks.

\subsubsection{Measuring Electrical Power}

Another way of measuring mass flow is by means of two precision platinum-resistance temperature sensors completely immersed into the flow stream. One sensor measures the temperature of the fluid, while the other is heated at a higher temperature than that of the fluid by means of an electronic control circuit (about $40{ }^{\circ} \mathrm{C}$ hotter). When the fluid begins to flow, the hotter sensor is cooled as the fluid passes taking heat away from it. The amount of heat removed is replaced by the electronic circuit so that the temperature of the hotter sensor is kept constant.

This method measures much higher mass flow rates that those previously explained because there is little pressure drop across the sensors (Fig. 5.25). The length $(L)$ of the sensors is of a few centimeters and the diameter $(D)$ of a few millimeters.

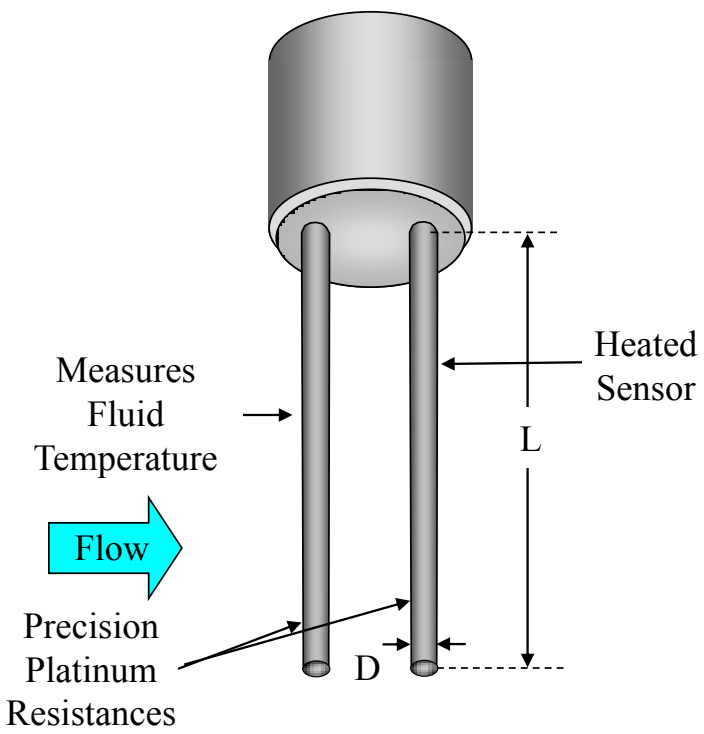

Fig. 5.25: Two precision temperature sensors are immersed into the flow stream. One sensor measures the temperature of the fluid and the other is heated. $D$ and $L$ are the sensor diameter and length respectively.

Assuming steady-state operation conditions, and disregarding heat losses by conduction and radiation, the power supplied by the heated sensor (Eq. (5.10)) equals the heat taken away by the fluid flow due to convective heat transfer (Eq. (5.9)), then 


$$
R I^{2}=h_{c} A\left(T_{2}-T_{1}\right)
$$

The left member in Eq. (5.12) is the electrical power supplied to the resistive sensor, which can be accurately measured, and the right member is the heat transferred from the heated sensor to the fluid due to natural and forced convection. In this case $A$ is constant $(A=\pi D L), D$ and $L$ being the sensor diameter and length respectively. The temperature difference is kept constant by the electronic circuit, but $h_{c}$ is variable and depends on the mass flow.

The mass flow of the fluid cannot be found analytically, but it can be known empirically by a calibration curve relating the power input to the mass flow passing by the sensor. Calibration has to be done with the same fluid in which the flowmeter will be used, and it should be performed in a pipe of similar characteristics. The calibration is stable as long as the coefficient $h_{\mathrm{c}}$ does not change with time.

\subsubsection{Hot Wire Anemometers}

Hot wire flowmeters (also called hot wire anemometers) are based on the measurement of the heat transferred from a heater to the environment that surrounds it. The sensor must be heated to a certain temperature greater than that of the environment. When the sensor is exposed to a fluid stream it loses heat by forced convection. This transfer increases with fluid velocity.

These flowmeters measure flow using the relation that exists between the electric power supplied to the heater and the velocity of the fluid in which it is immersed. Heat transfer from a resistive wire (probe) to the fluid in motion is given by

$$
A+B V^{n}=I_{s}^{2} R_{s}
$$

where $V$ is fluid speed, $A, B$ and $n$ are calibration constants, $I_{\mathrm{s}}$ is the electric current through the sensor and $R_{\mathrm{s}}$ is the sensor electric resistance. In some cases it may be assumed $n \approx 0.45$, but $n$ should be determined along with $A$ and $B$ by calibration. Assuming $R_{\mathrm{s}}$ invariable, in order to get these calibration constants, $I_{\mathrm{s}}$ must be measured for a number of known flow velocities and a least squares fit has to be performed for the values of the constants which produce the best fit to the data.

Therefore, once the relation between $V$ and $I_{s}$ has been established the flow speed can be estimated by measuring the electrical current $I_{s}$. This kind of anemometer is frequently used in wind tunnels and fluid mechanics laboratories because they are able to measure very fast fluctuations of fluid velocity.

It should be underlined that unlike thermal mass flowmeters, $I_{\mathrm{s}}$ is related to fluid velocity. If the velocity were measured inside a pipe, the pipe area $(A)$ should be known in order to compute the volumetric flow rate. If the fluid density $(\rho)$ is known, the mass flow can be computed from Eq. (5.14); but changes of density with temperature have to be taken into account to compute the mass flow rate correctly. 


$$
\frac{\Delta m}{\Delta t}=\rho V A
$$

Probes using this method are basically of two types: hot films and hot wires. The first are employed to measure in water and gases, and the last to measure in air.

\subsubsection{Hot Film}

Platinum films stuck on a quartz substrate are sometimes used as sensors (Fig. 5.26b) and are known as hot films. They are protected with alumina for use in gases and with quartz for use in liquids. They present an electrical resistance which is used to produce heat. The hot film has electrical contacts at the point where the wires are soldered to connect them to the electronic circuit. The size of these probes is small, for the example shown in the figure the diameter $D$ may be on the order of 0.06 to 0.15 $\mathrm{mm}$.

The film resistance $\left(R_{\mathrm{s}}\right)$ varies with temperature as

$$
R_{s}=R_{r}\left[1+\alpha\left(T_{s}-T_{r}\right)\right]
$$

where $R_{\mathrm{r}}$ is the resistance of the sensor at a reference temperature $T_{\mathrm{r}}$, and $\alpha$ is the temperature coefficient of the metal film resistance.

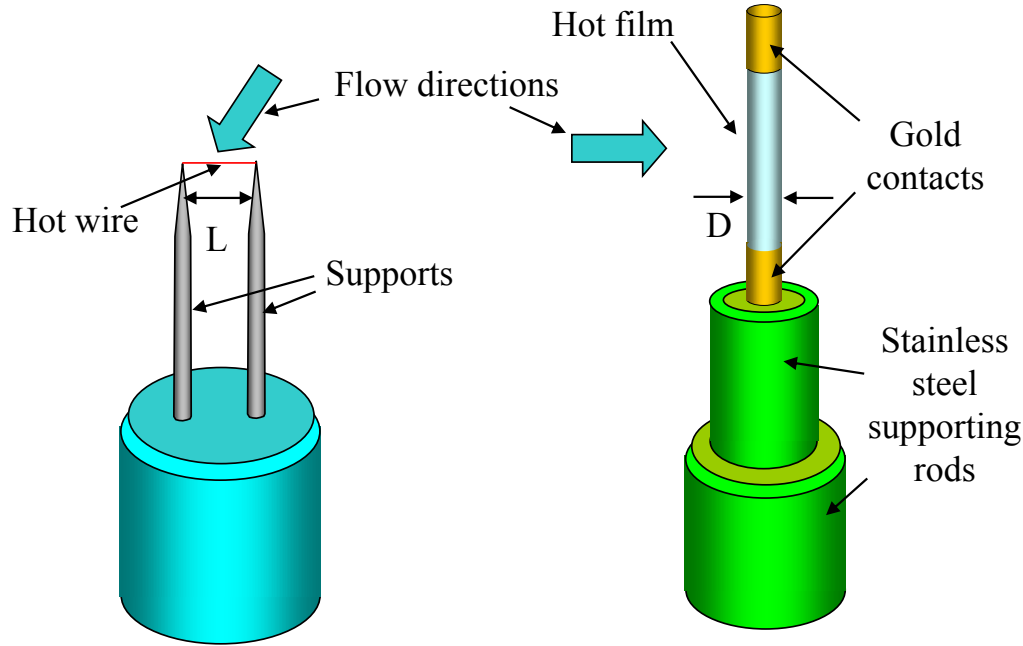

(a) (b)

Fig. 5.26: (a) A hot wire probe. (b) A hot film probe.

Usually, the sensor is part of a feed-backed Wheatstone bridge which keeps $T_{\mathrm{s}}$ constant, independently of the flow cooling the sensor. This is achieved by controlling 
the current through $R_{\mathrm{s}}$ such that $R_{\mathrm{s}}=$ constant. Then, measuring $I_{\mathrm{s}}$, the fluid velocity $V$ can be calculated from Eq. (5.13).

\subsubsection{Hot Wire}

It is another type of sensor used with the same operating principle but which has a different field of applications (Fig. 5.26a). Hot wire anemometry is an excellent tool for studying turbulent flow in air. It is useful to acquire velocity time series with high time and spatial resolutions. For these applications sensors are made of very small diameter wires. Probes are usually made of tungsten wires of a few millimeters in length and a few micrometers in diameter. Probes with $L=1 \mathrm{~mm}$ and $5 \mu \mathrm{m}$ in diameter, mounted on two needle-shaped prongs are reported (http://www.dantecdynamics. com). Because these devices and the associated electronic circuits respond very fast, the upper frequency of the transference bandwidth reaches $100 \mathrm{kHz}$.

These probes are sensitive to directional flow so that when two or three probes are orthogonally arranged they give information on the flow velocity components, but a directional calibration is required to determine the complete transfer of the instrument.

\subsection{Coriolis Mass Flowmeters}

\subsubsection{Introduction}

The Coriolis mass flow meter is perhaps one of the most anti intuitive measuring devices, and at the same time it is one of the most useful flowmeters. The fact that they measure mass flow directly is a characteristic offered by few flowmeters, and there are some applications where this kind of flowmeter is the most adequate option. If it were said that by measuring time it would be possible to estimate mass flow through a pipe, it would sound like a "magic trick". To some extent this instrument is the result of some wizard engineers which combines mechanical and electronic knowledge to produce a work of art. Therefore, to explain this ingenious device requires the use of several pages and a number of approximations. This explanation will follow the ideas found in the technical literature (Micro Motion Inc., 1996). The explanation requires beginning with the Coriolis force concept and how it appears. This concept is extensively developed in Section (3.10). 


\subsubsection{The Coriolis Flowmeter}

A flowmeter that uses the Coriolis force as its operating principle is shown in Figure 5.27a. The development of a simplified explanation will be based on the geometry shown in Figure 5.27b.

Figure 5.28 shows a simplification of the U-shaped tube of the flowmeter that is located on a plane which, for the sake of simplicity, is assumed to be horizontal. The plane is made to oscillate vertically by means of electromagnets. The physical principle by which the vibration is produced is similar to that shown for a tuning fork oscillator (Section (4.5.1)). In the flowmeter case the fluid-filled metal pipe represents the mass, and the elasticity of the metal, the spring. In practice the total vibration amplitude of the tube is small and the frequency low (e.g. $3 \mathrm{~mm}$ and $80 \mathrm{~Hz}$ ).
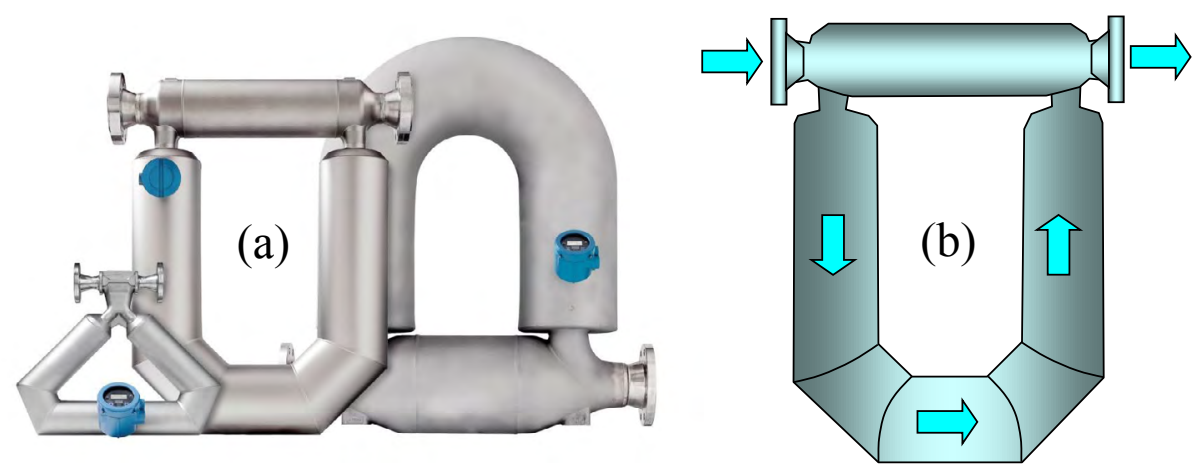

Fig. 5.27: (a) Coriolis Flowmeters. (Courtesy of Emerson Process Management - Micro Motion, Inc.). (b) Schematic of the flowmeter; arrows indicate flow path.

We will follow an intuitive introduction to the phenomenon to develop the working principle. At the beginning no flow circulates and the tube vibrates as in Figure 5.28a. It must be recalled that the flow passing through the fixed portion of the tube (Rotation Axis) has a certain momentum. Suppose that in passing the curve, when the fluid is in the first arm of the " $U$ " tube, the " $U$ " tube is moving upwards, so that the fluid is forced to acquire the vertical movement of the vibrating tube. The fluid is thus compelled to change its momentum, and because it refuses to be moved upwards, it produces a thrust down onto the wall of the tube Figure 5.28b. When the fluid is in the opposite arm of the " $U$ " tube it is forced to decrease its vertical momentum and it resists pushing the tube up. These two forces in opposite directions produce a torque on the tube.

It is interesting to note that in the part of the tube that is parallel to the axis of rotation, $\mathbf{V}$ is collinear with $\boldsymbol{\omega}(\sin \theta=0)$, so on that portion of the tube there is no 
Coriolis force when the " $U$ " is horizontal. At the central point there is a node of the twisting motion.

The amount that the tube is twisted depends on the mass flow. Applying Eq. (3.31) and the rule of the corkscrew for the vector product, the directions of the Coriolis forces are obtained and displayed in Figure 5.28b along with the torque. Obviously, when the tube stops moving upwards and begins to move down, the direction of the torque is reversed.
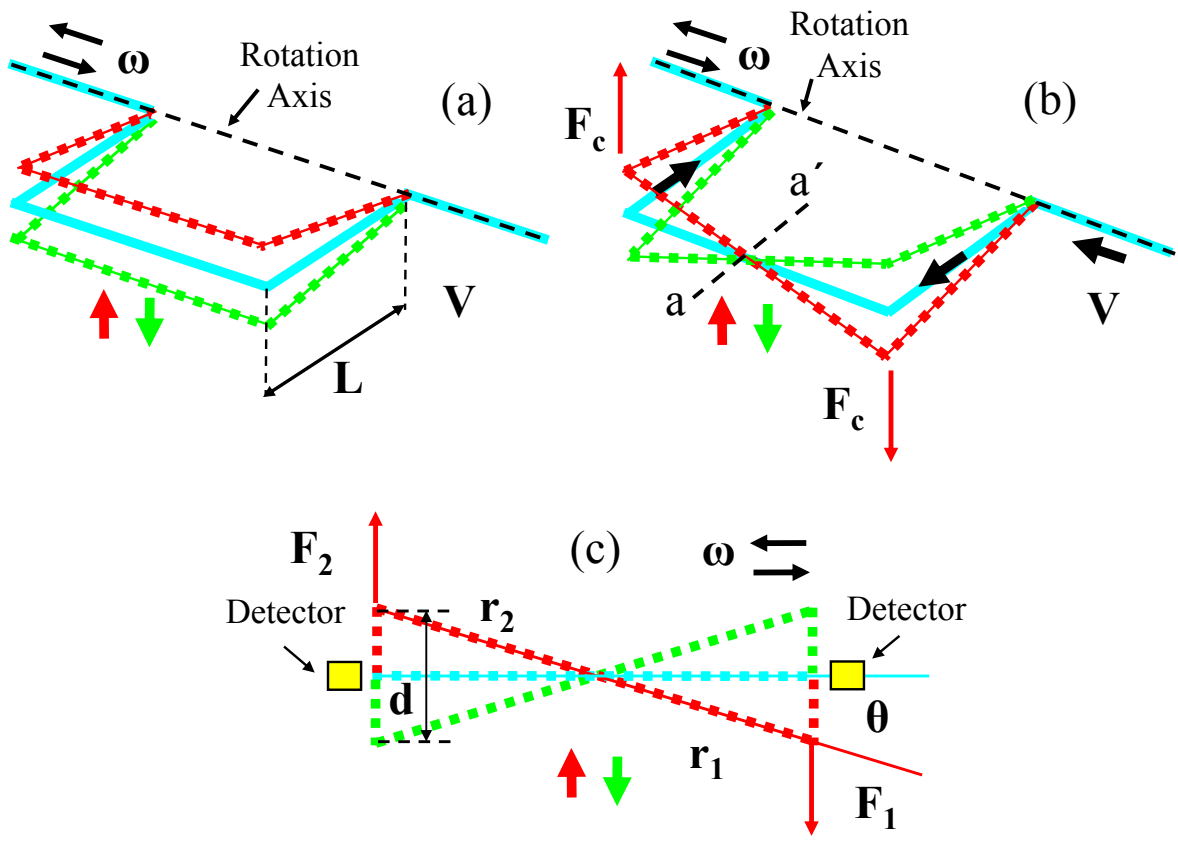

Fig. 5.28: (a) The $U$ tube is horizontally placed and vibrated up and down about the rotation axis. Flow is null. (b) Flow circulates as indicated by the arrows; forces $F_{c}$ appear. (c) Forces $F_{c}$ create an oscillating momentum. The detectors permit the difference in time between the tubes to be known.

Some relationships based on the Coriolis force (Eq. (3.31)) will be developed to show that the mass flow can be measured with this device. Several simplifications are made for this purpose. The oscillating movement up and down, which is an accelerated movement, has to be assumed as a rotating motion of constant angular velocity value $(\omega)$ whose axis of rotation is the fixed part of the pipe (Figs. 5.28a and 5.28b). Therefore, the direction of the vector $\boldsymbol{\omega}$ agrees with the rotation axis. Also, it has to be assumed that the velocity $\mathbf{V}$ of the fluid has the direction of the pipe in each section of the " $U$ " tube; and its direction is as depicted in Figure 5.28b. 
Because of the symmetry of the tube, (Figure 5.28c), the forces $\mathbf{F}_{1}$ and $\mathbf{F}_{2}$ exerted by the fluid in each of the branches are equal in magnitude but opposite in direction. When the tube vibrates, the forces create an oscillating momentum $\mathbf{M}$ around the axis a-a', with radii $\mathbf{r}_{1}=\mathbf{r}_{2}=\mathbf{r}$ approximately given by Eq. (5.16), because the forces and the radii are nearly perpendicular.

$$
M \approx r_{1} F_{1}+r_{2} F_{2}
$$

From Eq. (3.31), and taking into account that the Coriolis forces $F_{1}$ and $F_{2}$, and the distances $r_{1}$ and $r_{2}$ are equal, we get

$$
M=2 r F=4 m V \omega r
$$

As we are interested in the magnitude of the Coriolis force we will disregard the negative sign in Eq. (3.31). Then, due to the mass flow rate passing through the tube and the rotation imposed by the vertical vibration, there appears an oscillating momentum applied to the tube. It should be noted that the momentum is proportional to the mass ( $m=\rho \mathrm{Vol}, \rho$ being the fluid density and $\mathrm{Vol}$ the fluid volume).

It is interesting to note from Eq. (5.17) that once the instrument is built (i.e. $\boldsymbol{\omega}$ and $\mathbf{r}$ being fixed), and for a given fluid velocity $V$, the measured momentum depends only on the mass of the fluid. This means that the instrument will produce lower momentum with low density fluids, resulting less sensitive for this kind of fluid.

The speed $\mathbf{V}$ is replaced by the distance $L$ traveled by the fluid in time $t$. The mass flow rate $Q_{\mathrm{m}}$ is defined as the mass $m$ that passes through a given section of the pipe in time $t$, thus Eq. (5.17) is rewritten as

$$
M=4 m \omega r \frac{L}{t}=4 L \omega r \frac{m}{t}=4 L \omega r Q_{m}
$$

Figure 5.28c shows that the momentum $\mathbf{M}$, which is proportional to mass flow rate, causes an angular deflection $\theta$ about the axis a - $\mathrm{a}^{\prime}$. A resistant torque $\mathbf{T}$ due to the elasticity of the tube $\left(k_{\mathrm{s}}\right)$ opposes to $\mathbf{M}$. Equating both torques and solving for the mass flow rate, we get

$$
T=k_{s} \theta=M=4 L \omega r Q_{m} ; \quad Q_{m}=\frac{k_{s} \theta}{4 L \omega r}
$$

This equation states that the mass flow can be known if the angular deviation $\theta$ is known. For this purpose two detectors are placed as in Figure 5.28c. Each detector measures the instant at which each tube arm passes by the midpoint of the swing.

When the liquid is at rest, there is no bending of the tube and both arms pass by the midpoint at the same instant (Fig. 5.28a). As the fluid speed increases, the angle $\theta$ begins to increase, which increases the time difference $(\Delta t)$ between the crossings of both arms of the tube by the detector's positions. It is possible to relate the distance traveled by the extreme of the tube $(d)$ with the angle $\theta$. This distance can be estimated from the average tangential velocity of the extreme of the tube $\left(v_{t}\right)$ and $\Delta t$. For small $\theta$, $\sin \theta \approx \theta$ and $v_{\mathrm{t}}=\omega L$, 


$$
\sin \theta \approx \theta=\frac{d}{2 r}=\frac{v_{t} \Delta t}{2 r}=\frac{\omega L \Delta t}{2 r}
$$

From Eqs. (5.19) and (5.20),

$$
Q_{m}=\frac{k_{s} \Delta t}{8 r^{2}}
$$

Expressed in words, Eq. (5.21) states that the mass flow is proportional to the constructive constants and to the time interval $\Delta t$, and is independent of the vibration frequency.

All this simplified mathematics was done to show that in order to measure the mass flow it is only needed to know the time difference $\Delta t$. In practice this time difference is measured by means of magnetic detectors placed as in Figures 5.28c and 5.29.

Figure 5.29 shows a schematic diagram of the instrument; it depicts a real flowmeter in which the flow is distributed into two vibrating tubes. The theory remains the same for each tube, but flow splitting has some practical advantages. One advantage is that the external vibration (due, for example, to the pumps that impel the fluid in the main pipe) which moves both tubes in a similar way is a common mode noise, affecting both measures in the same way. Thus, measuring the relative motion between both " $U$ " tubes, known as a differential signal, greatly attenuates the common noise introduced by the external vibrations. The figure also shows the location of the driver, used to produce the vibration of both tubes, and the detectors, used to measure the time difference $\Delta t$.

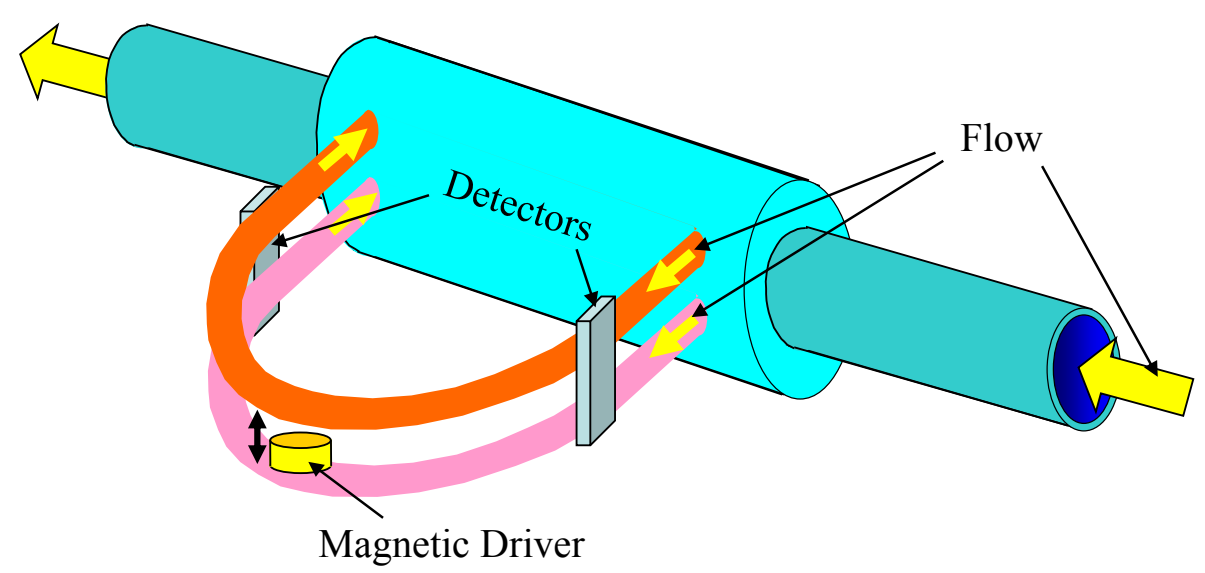

Fig. 5.29: The flow to be measured is driven into two vibrating tubes. The magnetic driver makes the tubes vibrate $180^{\circ}$ out of phase. Detectors measure the differential time between crossings of both arms of each tube. 


\subsubsection{Some Characteristics of Coriolis Flowmeters}

This type of flowmeter is not affected by changes in the fluid temperature, pressure, viscosity, density or velocity profile. It is also insensitive to inlays that change the internal section of the tube. They can be used in almost any type of fluid: mud, multiphase fluids, dry gases, steam, pulsating flow, etc. In the case of gases, their densities must be sufficiently high so that the produced torque is detectable. They are manufactured for several pipe diameters. The errors are less than $\pm 0.2 \%$ for dynamic ranges of 1: 25 or greater.

As disadvantages it should be mentioned that they produce moderate load losses and are large in size compared to other technologies (e.g. electromagnetic). Since the flow is separated into two tubes of smaller sections than the inlet pipe, cavitation or secondary phases could be generated which can clog the tubes, interrupting the measurements.

\subsubsection{Measuring Density}

Each tube of the flowmeter may be considered as a mass and spring set which can vibrates at a certain resonant frequency (Section (4.5.1)). This frequency depends on the mass of the tube plus the mass of the fluid, as well as on the tube elasticity. In order to bring the tube to its resonant frequency, a driving coil and a feedback circuit is used and then the tube always oscillates at resonance.

The mass of fluid filling the pipe depends on the fluid density and the internal volume of the pipe $(m=\rho \mathrm{Vol})$. Let us assume the internal volume of the tube, the tube's elasticity and the tube mass to be constant. Then the resonant frequency will depend only on the fluid density. Thus knowing the resonant frequency of the tube the density of the fluid may be inferred.

The frequency at resonance can be measured using the same detectors employed to measure the mass flow. To compensate for changes in the elastic modulus of the pipe's material due to temperature changes, a temperature sensor is used to correct density measurements.

\section{References}

McMahon, M. \& Rouse, S. (2008). A Tale of Two Thermals: Capillary \& Immersible. Core Technology White Paper Series, Part 1: Thermal. Sierra Instruments.

Micro Motion Inc. (1996). Micro Motion Mass Flow Meter Manual, Boulder, CO, USA.

Miller R.W. (1996). Flow Measurement Engineering Handbook. New York: McGraw-Hill.

Terray, E. A., Brumley, B. H., \& Strong, B. (1999). Measuring waves and currents with an upward-looking ADCP. Proc. Sixth Working Conf. on Current Measurements, San Diego, CA, IEEE (pp. 66-71).

A Tale of Two Thermals: Capillary \& Immersible. Core Technology White Paper Series. Sierra Instruments. Available at: http://sierratechsupport.com/news_letter/newstrak/a_tale_of_two_ thermals.pdf

http://www.dantecdynamics.com/Default.aspx?ID=1057 